
\title{
$\angle$ Research Square \\ Exosomal miR-331 from Chemoresistant Osteosarcoma Cells Induced Chemoresistance Through Autophagy
}

\section{Aiqing Zhao}

The First Affiliated Hospital of Inner Mongolia Medical University

\section{Yan bin Zhao}

Healthcare Center of Ning Bo Customs

Wanlin Liu

The Second Affiliated Hospital of Inner MongoliaMedical University

\section{Wei Feng}

The Second Affiliated Hospital of Inner Mongolia Medical University

\section{Wenhua Xing}

The Second Hospital of Inner Monglia Medical University

\section{Zhenqun Zhao}

The Second Hospital of Inner Mongolia Medical University

\section{Shuxia Cui}

The Second Hospital of Inner Mongolia Medical University

\section{Rui Bai ( $\nabla$ bairuiandaiqing@126.com )}

The Second Affiliated Hospital of Inner Mongolia medical university

\section{Chenyang Meng}

The Second Affiliated Hospital of Inner Mongolia Medical University

\section{Research}

Keywords: Osteosarcoma, chemoresistance, microRNA-331, autophagy

Posted Date: June 16th, 2021

DOl: https://doi.org/10.21203/rs.3.rs-587973/v1

License: (c) (1) This work is licensed under a Creative Commons Attribution 4.0 International License. Read Full License 


\section{Abstract \\ Background}

Osteosarcoma (OS) is a highly malignant tumor. Improving chemotherapeutic resistance is very important to improve the survival rate of OS. Exosomes and microRNAs (MiRNA) play important roles in the mechanism of chemotherapeutic resistance transmission. More and more researches focus the mechanism of miRNAs carried by exosomes in the transmission of chemotherapeutic resistance of OS. This study focused on exploring the mechanism of exosomal miR-331 in the transmission of chemoresistance in OS.

\section{Methods}

We cultured OS drug-resistant cells and extracted exosomes of these cells. The secretion and uptake of exosomes in OS drug-resistant cells and OS cells (OSCs) were confirmed by fluorescence tracking assay and transwell experiments. The differential expression of microRNA-331 (miR-331) in exosomes of OS resistant and OS cells was investigated by RT-PCR. The effects of drug-resistant exosomes on proliferation and migration of OS cells were determined by MTT assay and scratches assay. MDC staining, RT-PCR, and Western blot were used to detect the role of autophagy which regulated by drugresistant cell-derived exosom-miR-331.

\section{Results}

We found that the expression difference of miR-331 between drug-resistant cells of MG63 and HOS cell lines and tumor cells was the most significant. Drug resistant OSCs secreted exosomes and were ingested by OSCs, which then promoted OSCs to acquire drug resistance. In addition, exosomes secreted by drug-resistant OSCs promote drug resistance by carrying miRNAs. Interestingly, inhibition of miRNA resulted in reduced drug resistance transmission of exosomes. Finally, we found that the exosomes secreted by drug-resistant OSCs could induce autophagy of OSCs by carrying miR-331, thus making OSCs acquire drug resistance. Inhibition of miR-331 can effectively improve drug resistance of OSCs.

\section{Conclusions}

Chemoresistant OSCs-derived exosomes promote the transmission of drug resistance by carrying miR331 and inducing autophagy. Inhibition of miR-331 could effectively alleviate drug resistance of OSCs.

\section{Background}


Osteosarcoma (OS) is a common primary malignant tumor in adolescent and elderly people ${ }^{1}$. Besides,OS is one of the commonest primary malignant tumors of bone ${ }^{1}$. It metastasizes fast, and has a high death rate $^{1}$. Guideline for the treatment of OS is surgery combined with chemotherapy ${ }^{2}$. It has been reported that the combination of three types of chemotherapy, including methotrexate, doxorubicin and cisplatin (CDDP), has achieved a good outcome, particularly for children and young adults ${ }^{3}$.

Johnstone identified an extracellular vesicle called exosome which involved in reticulocyte transferrin receptor transport ${ }^{4,5}$. Exosomes play an important role in transport of intercellular material ${ }^{5}$. Active tumor cell could secrete more exosomes into the recipient cell and the recipient cells could not only be normal cells but tumor cells ${ }^{6}$. Exosome could lead normal cell transfer to tumor cell ${ }^{6}$. Besides, exosome could bring some bioactive molecule and cytokines so that it could regulate signal pathway of recipient cells, further to regulate the invasion, proliferation and chemosensitivity of tumor cell ${ }^{7-9}$. A lot of microRNAs (miRNAs) could be carried by exosome ${ }^{10}$. MiRNA could directly target the $3^{\prime}$ UTR of target gene mRNA, further to suppress the expression of target gene and to regulate the protein expression of target gene ${ }^{11}$. It was well-known that miRNA play significant role in multiple metabolism of cells. It has been found that miRNA could regulate the invasion, proliferation and chemosensitivity of tumor cell. The chemosensitivity of tumor cell could be regulated by damaging DNA, apoptosis, autophagy and regulation of signal pathway ${ }^{12}$.

Current studies on exosome-miRNA mainly involve bone marrow mesenchymal stem cells(BMSCs). It has been found that BMSCs could derive exosome to regulate osteosarcoma cell (OSC) proliferation through the Hedgehog signaling pathway ${ }^{13}$.Studies have shown that drug-resistant OS cells can carry multiple drug-resistant gene phenotypes into non-drug-resistant cells through exosomes, which causing non-drugresistant OSC to develop drug resistance ${ }^{14}$.

It has been reported that miR-331-3p might lead to poor outcome of tumor. However, there was few study on association between miR-331 and OS. Autophagy was found highly associated with drug resistance of tumor. The aim of this study was to investigate the relationship between exosome-miR-331 and OS. Further to explore the whether miR-331 could regulate the drug resistance of tumor by autophagy in OS.

\section{Materials And Methods}

\section{Culture of OSC}

MG63, HOS, U2OS, Saos2 cells were resuscitated and cultured in MEM medium containing $10 \%$ fetal bovine serum (100 U/ mL penicillin, $100 \mu \mathrm{g} / \mathrm{mL}$ streptomycin) in a cell incubator at $37^{\circ} \mathrm{C}$ and $5 \% \mathrm{CO} 2$. After $80 \%$ of the cells converged, the medium was removed and PBS was added for washing once or twice. After removing PBS, $1 \mathrm{~mL}$ trypsin was added for digestion for 1-3 min, and $3 \mathrm{~mL}$ of the complete medium was added to neutralize trypsin and stop digestion. The digested cells were transferred to a 15 
$\mathrm{mL}$ centrifuge tube and centrifuged at 1000 RPM for 5 min. After the supernatant was dumped, the cells were added to the $3 \mathrm{~mL}$ medium to resuspend, and the cells were subcultured in a petri dish at 1:3.

\section{Culture of cisplatin resistant OSC}

When the OSC grew to the logarithmic stage, $0.1 \mathrm{mg} / \mathrm{L}$ cisplatin (CDDP) was added to the culture medium. After $24 \mathrm{~h}$, the culture medium without CDDP was replaced. After the stable growth of cells, the passage was carried out. The above method was used for continuous treatment for 5 times, and the CDDP concentration was changed to $0.2 \mathrm{mg} / \mathrm{L}$ for 5 times, and the CDDP concentration was changed to $0.5 \mathrm{mg} / \mathrm{L}$ for 3 times. After a total of 190 days of induction, OSC could grow stably and pass normally under the condition of $0.5 \mathrm{mg} / \mathrm{L}$ CDDP, indicating that the cell line could tolerate $0.5 \mathrm{mg} / \mathrm{L}$ CDDP, and the drug-resistant strain was named OSC/CDDP.

Reverse transcription-polymerase chain reaction (RT-PCR)

\section{Screening of OS cell lines and detection of miR-331 expression}

By measuring the expression of miR-331 in OS cell lines through RT-PCR, OS cell lines to be studied were selected.

Direct Zol RNA microPrep Intracellular RNA was extracted in the toolkit and exosome RNA was extracted through the miRCUTTE RNA isolation toolkit. The cDNA was synthesized by SuperScript III and TaqMan through 200ng isolated RNA The ABI Prism 7500 sequence detection system was amplified.2- $\triangle \Delta \mathrm{CT}$ method was used to determine the value mRNA expression of the corresponding miRNA or gene (miRNA should take U6 as internal reference)

\section{Cell transfection}

$24 \mathrm{~h}$ before OSC transfection, the cells were digested with trypsin, and the concentration was adjusted to $2 \times 10^{5}$ cells/ mL, and the cells were inoculated in 6-well culture plates. After the cells were bonded, they were cultured in $2 \mathrm{~mL}$ DMEM medium containing serum and without antibiotics. During transfection, the mimics (miR-199a mimics) were diluted with $200 \mu \mathrm{L}$ Opti-MEM medium, mixed gently, and stood for 5 min at room temperature. At the same time, $5 \mu \mathrm{L}$ Lipofectamine 2000 was diluted with $200 \mu \mathrm{L}$ Opti-Mem I medium, mixed gently, and stood for 5 min at room temperature. The diluted mimics (miR-199a mimics) and Lipofectamine 2000 were mixed and let stand for $25 \mathrm{~min}$ at room temperature, then added into the cell culture well and shaken evenly. Cells were placed in an incubator at $37^{\circ} \mathrm{C}$ with $5 \% \mathrm{CO} 2 \mathrm{for} 30 \mathrm{~h}$, and then transferred to 3 culture plates with a diameter of $10 \mathrm{~cm}$. After transfection, the next experiment was carried out, that is, the expression level of miR-199a in cells was detected by qRT-PCR $48 \mathrm{~h}$ after culture.

\section{Exosome extraction}

When OSC grew to 70-75\% convergence, the original culture medium was discarded and washed with PBS buffer for 3 times. Serum-free medium was added for further culture for $36 \sim 48 \mathrm{~h}$. The culture 
medium was collected and centrifuged at $4^{\circ} \mathrm{C}$ for $300 \times \mathrm{g}$ for $10 \mathrm{~min}$ to remove the remaining cells, followed by centrifugation at $4{ }^{\circ} \mathrm{C}$ for $20 \mathrm{~min}$ at $2000 \times \mathrm{g}$ to remove the cell fragments, and centrifugation at $4{ }^{\circ} \mathrm{C}$ for $45 \mathrm{~min}$ at $11,000 \times \mathrm{g}$ to further remove the impurities and retain the supernatant. The supernatant was centrifuged at $110,000 \times \mathrm{g}$ at $4^{\circ} \mathrm{C}$ for $90 \mathrm{~min}$, then the supernatant was discarded, $1 \mathrm{~mL}$ of $1 \times$ PBS was added to each centrifuge tube to resuspend the precipitation, and the suspension in each tube was collected in a centrifuge tube, and then centrifuged at $110,000 \times \mathrm{g}$ at $4^{\circ} \mathrm{C}$ for $70 \mathrm{~min}$. Discard the supernatant and resuspend the precipitation with $100 \mu \mathrm{L} 1 \times$ PBS.

\section{PKH26 labeled exosomes}

$100 \mu \mathrm{L}$ of $100 \mu \mathrm{g} / \mathrm{mL}$ of exogenous body weight was suspended in $1 \mathrm{~mL}$ of PBS, then $4 \mu \mathrm{L}$ of PKH26 fluorescent dye solution was added and incubated at $37^{\circ} \mathrm{C}$ for $20 \mathrm{~min}$. Centrifuge at $100,000 \times \mathrm{g}$ for 70 min, discard the supernatant, suspend the exogenous body weight in $10 \mathrm{~mL} \mathrm{PBS}$, and centrifuge at $100,000 \times \mathrm{g}$ at $4{ }^{\circ} \mathrm{C}$ for $70 \mathrm{~min}$. The excess dye was removed, supernatant was discarded, and the exogenous body weight was suspended in $100 \mu \mathrm{L}$ PBS for reserve. The labeled PKH26 and OSC were cultured in MEM medium containing $10 \%$ fetal bovine serum $(100 \mathrm{U} / \mathrm{mL}$ penicillin, $100 \mathrm{ug} / \mathrm{mL}$ streptomycin) in a cell incubator at $37^{\circ} \mathrm{C}$ and $5 \% \mathrm{CO} 2$ for $12 \mathrm{~h}$. The medium was removed, the cells were washed twice with PBS, fixed with $4 \%$ paraformaldehyde and stained with DAPI. The cells were observed with red $\mathrm{PKH} 26$ under confocal fluorescence microscope.

\section{Transwell assay}

Cy3-miR-199a mimic was transfected into OSC/CDDP cells. After that, OSC /CDDP ( $1 \times 10^{6} /$ well) and OSCwere co-cultured at a 1:1 ratio on trans-well plate for $12 \mathrm{~h}$. The upper compartment was OSC/CDDP, the lower compartment was OSC. In the control group, only CY3 was transfected without miR-199a. After rinsing the MG63 twice with PBS, the fluorescence of Cy3 in the OSC was observed under confocal microscope.

\section{Verification of exosome function}

Exosomes extracted from OSC/CDDP and OSC were divided into three groups: drug-resistant exosome group (Exo/CDDP), OSC exosome group (Exo/S), and blank control group PBS (PBS). The exosomes (2 $\mu \mathrm{g})$ were added into OSC $\left(1 \times 10^{6} /\right.$ well) to establish the experimental group. After co-culture for 12 hours, $1-60 \mathrm{ng} / \mathrm{mL}$ CDDP (4 gradients of 1, 10, 30 and 60) was added to the three groups, respectively, for 24 hours, and then the cell activity was detected by MTT. The migration ability was detected by scratch test, which was divided into four groups: MG63, MG63 + ExO /S, MG63 + ExO /CDDP, and MG63/CDDP. The exosomes $(2 \mu \mathrm{g})$ were co-cultured with cells $(1 \times 106 /$ well $)$ for 12 hours, and then the wound healing assay was performed.

\section{MTT assay}

After CDDP treatment, $10 \mu \mathrm{L}$ MTT was added to each well for $3 \mathrm{~h}$, a-MEM was removed from the complete medium, $150 \mu \mathrm{L}$ dimethyl sulfoxide was added to each well and shaken evenly. The absorbance was measured at $570 \mathrm{~nm}$ by enzyme linked immunoassay. 


\section{Wound Healing assay}

The OSCs were scraped with a pipet of $200 \mu \mathrm{L}$. Photos were taken at $\mathrm{Oh}$ and $24 \mathrm{~h}$. The distance from the damaged area to the disappearance at $0 \mathrm{~h}$ was used as the quantification and relative invasion rate of remodeling.

\section{Verification of miRNA carrying function in exosomes}

The cells were divided into three groups: OSC/CDDP, OSC + Exo/CDDP, OSC + Exo/CDDP + GW4869 (10 $\mu \mathrm{m}$, cultured for $24 \mathrm{~h}$ ), the expression of miR-199a was detected by RT-PCR. Identifying the key factors for exosomes to transport miRNAs from OSC/CDDP. Then, by knocking out the Drosha protein of MG63/CDDP, the activity of exosomal-miRNA was inhibited. The expression of miR-331 and miR-199a in exosomes was detected by RT-PCR to determine whether miRNA was inhibited by knocking out the Drosha protein. Then, the exosomes of OSC/CDDP were extracted and co-cultured with OSC. The exosomes were divided into three groups: OSC + Exo/CDDP (treated with Drosha), OSC + Exo/CDDP (not treated with Drosha) and OSC + PBS. After 12 hours of culture,2 $\mu \mathrm{m}$ CDDP was added to each well for MTT detection.

\section{The MDC staining}

Cells of each group were mixed and cultured with $\mathrm{MDC}$ at $37^{\circ} \mathrm{C}$ and room temperature. The IOD value was then determined under a confocal fluorescence microscope using anti-fluorescence quenching slides and DAPI staining.

\section{Western-bolting}

Ice lysis of cells or exosomes in RIPA lysates containing protease inhibitors. Equal amounts of total proteins between different groups were separated in SDS-PAGE gel $(100 \mathrm{~V}, 1.5 \mathrm{~h})$ and transferred to 0.22 $\mu \mathrm{m}$ polyethylenedifluoride (PVDF) membrane at $(280 \mathrm{~mA}, 1.5 \mathrm{~h})$. Then it was treated with $5 \%$ skim milk powder washing buffer at room temperature for 1 hour and added with primary antibody at $4{ }^{\circ} \mathrm{C}$ overnight.On the second day, the membrane was cleaned with TBST and incubated with secondary antibody, and each band was detected using an enhanced chemiluminescence kit.

\section{Inoculation of tumor cells}

OSCs were cultured in MEM medium containing 20\% fetal bovine serum, $100 \mathrm{U} / \mathrm{mL}$ penicillin, $100 \mu \mathrm{g} /$ $\mathrm{mL}$ streptomycin, and cultured in an incubator containing $5 \% \mathrm{CO}_{2}$ at $37^{\circ} \mathrm{C} .80 \% \sim 90 \%$ of the cells growing in the culture dish were digested with trypsin, centrifuged at 1000 RPM, supernatant was removed, the cells were washed by adding PBS, and then resuspend by adding PBS again. Cells were counted and the number was adjusted to $2 \times 10^{6} \mathrm{cells} / \mathrm{mL}$. According to the number of nude mice, a sufficient amount of cell suspension was prepared for use when inoculating nude mice. The prepared cell suspension was stored in a refrigerator at $4^{\circ} \mathrm{C}$ and inoculated into nude mice as soon as possible. The injection site was uniformly $0.3 \mathrm{~cm}$ from the right forearm armpit of the nude mice on the back. After iodine volt cotton ball disinfection, a $1 \mathrm{~mL}$ syringe was used to extract $0.2 \mathrm{~mL}$ of the mixed cell 
suspension (density: $2 \times 10^{6} / \mathrm{mL}$ ). After the needle of the syringe penetrated the skin of the nude mice, the needle was slightly picked up, moved to the injection site, and the cell suspension was injected slowly. After the injection, the sterile cotton ball is pressed into the needle, and the experimental instruments and waste are disposed of innocently. The newly modeled nude mice were put into cages with new padding, and the mental state, activity and feeding condition of the nude mice, and whether there was redness and swelling at the injection site were observed the next day.

\section{Grouping and drug intervention}

When nodules appeared in nude mice (about 7 days after inoculation), the NS group was intraperitoneally injected with $100 \mu \mathrm{L}$ of normal saline twice a week for 5 weeks. CDDP group was intraperitoneally injected with CDDP (2 mg/kg/ time) twice a week for 5 weeks. Specific groups are as follows:MG63 group ( $n=6), M G 63+$ Exo group $(n=6), M G 63+$ Exo/CDDP group $(n=6), M G 63+$ Exo/CDDP + miR-331-3p inhibitor group $(n=6), M G 63 / \operatorname{CDDP}$ group $(n=6)$.

\section{Detection indexes}

Animal observation: the body weight, mental state, excitement, biting and activity of the nude mice were recorded. Tumor measurement: After tumor formation was visible to the naked eye, the transplanted tumor was measured with a ververier caliper to determine the short diameter $(\mathrm{W})$ and long diameter $(\mathrm{L})$. The tumor volume was calculated according to the formula $V(\mathrm{~cm} 3)=L \times W 2 / 2$, and the tumor size was measured twice a week. The final sampling: 6 weeks after tumor grafting in nude mice, tumor tissue was collected, the volume and weight of tumor were calculated, fixation with $4 \%$ paraformaldehyde.

\section{Immunohistochemistry}

The obtained tissues were embedded in paraffin sections, sealed with endogenous peroxidase, antigen repair, exposed to antigen determinants, and incubated with primary and secondary antibodies. DAB staining was followed by redyeing, dehydration, transparency, sealing and observation under light microscope.

\section{Statistical analysis}

Each experiment was performed three times and the results are shown as the mean \pm standard deviation. SPSS 23.0 software (SPSS, Inc.) was used for statistical analysis. Differences among three or more groups were compared by one-way analysis of variance (ANOVA); Tukey's test was used form comparisons of data between two groups. $P<0.05$ was considered, to indicate a statistically significant difference.

\section{Result}

\section{The expression of miR-331 in each cell line}


The expression of miR-331 in OS cell lines were higher than that in osteoblastic cell line. The expression of miR-331 were highest in MG63 cell line compared with that in other OS cell lines (figure.1). In thus, we used MG63 as study cell lines for further study.

\section{Identification of exosomes}

\section{Flow cytometry}

Flow cytometry was used to analyze the expression of CD63 and CD81 surface proteins in the supernatant exosomes of the MG63 and MG63/CDDP cells. The positive rates of CD63 in the supernatant exosomes of the MG63 and MG63/CDDP cells were $51.2 \%$ and $40.4 \%$, respectively. The positive rate of CD81 was $35.1 \%$ and $42.5 \%$, respectively (Fig. 2 ).

\section{Granularmetric analysis}

The particle distribution coefficients of the detected samples MG63 and MG63/CDDP were between 0.09 and 0.8 , which proved that the dispersion of the collected exosomes was moderate and the confidence of the detection results was high. The particle sizes of MG-63 and MG-63/CDDP samples ranged from 30 $\mathrm{nm}$ to $150 \mathrm{~nm}$, accounting for $90.2 \%$ and $92.1 \%$, respectively (figure.3).

\section{Transmission electron microscopy of exosomes}

Electron microscopy of exosomes of MG63 and MG63/CDDP were shown in figure.4.

\section{Exosome secretion and transport}

PHK26 labeled exosomes from MG63/CDDP cell line could be absorbed by MG63 cell line. According to the transwell experiment and RT-PCR, Cy3 and miR-199a from MG63/CDDP could be transferred into MG63 (Figure.5 and figure.6).

\section{Function of exosomes and miRNA carried by exosome}

At the concentrations of $10 \mathrm{ng}, 30 \mathrm{ng}$ and $60 \mathrm{ng}$ of cisplatin, MG63 proliferation was enhanced after treated by exosomes from MG63/CDDP according to the MTT assay (figure.7A). Besides, exosomes from MG63/CDDP transfected by siRNA-Drosha could not enhance proliferation of MG63 (figure.7B). The exosomes from MG63/CDDP treated by GW4869 could lead the low expression of miR-199a in MG63 compared with MG63/CDDP while MG63 treated with exosomes from MG63/CDDP could promote the expression of miR-199a compared with MG63/CDDP (Figure.8). The ability of MG63 to migrate was also enhanced after treated by exosomes from MG63/CDDP according to the wound healing test (figure.9).

\section{Exosomes from MG63/CDDP could induce autophagy of MG63.}

According to the MDC staining, mean fluorescence intensity in group MG63/CDDP was higher than that in group MG63 while exosomes from MG63/CDDP could promote the mean fluorescence intensity of 
MG63(figure.10). The expression of LC3 was higher in group MG63 + exosomes from MG63/CDDP than that in group MG63 while expression of p62 was lower in group MG63 + exosomes from MG63/CDDP than that in group MG63 according to the RT-PCR and western bolting (figure.11).

\section{MiR-331 carried by exosomes could promote the autophagy to induce drug resistance in OS}

The cell proliferation of group MG63/CDDP was the highest and the cell proliferation of MG63 could be improved after treated by exosomes from MG63/CDDP. The cell proliferation of MG63 treated by exosomes from MG63/CDDP which transfected by miR-331-3p inhibitor was reduced compared with MG63 treated by exosomes from MG63/CDDP without transfecting miR-331-3p inhibitor (figure.12).

The expression of LC3 was improved in MG63 after treated by exosomes from MG63/CDDP while the expression of LC3 was inhibited in MG63 after treated by exosome from MG63/CDDP which transfected by miR-331-3p inhibitor compared with MG63 treated by exosomes from MG63/CDDP without transfecting miR-331-3p inhibitor. Besides, the expression of p62 was reduced in MG63 after treated by exosomes from MG63/CDDP while the expression of p62 was promoted in MG63 after treated by exosome from MG63/CDDP which transfected by miR-331-3p inhibitor compared with MG63 treated by exosomes from MG63/CDDP without transfecting miR-331-3p inhibitor (figure.13).

\section{MiR-331 carried by exosomes from MG63/CDDP promote tumor growth and the anti-proliferative effect of CDDP.}

In Normal Saline group, the tumor volume was biggest in the group of MG63/CDDP and the tumor volume in MG63 + Exo/CDDP group was bigger than that in MG63 and MG63 + Exo group. Besides, the tumor volume in MG63 + Exo/CDDP + miR-331-3p inhibitor group was smaller than that in MG63 + Exo/CDDP group. In CDDP treatment group, the results were consistent with the normal saline group which suggested that miR-331-3p inhibitor could decrease the resistance of CDDP in OS (figure.14).

\section{Discussion}

In this study, we found that the transmission role of miRNA-exosome in drug resistance in OS. The chemoresistance of OSC could be transferred through exosome which derived from chemoresistant OSC. It has been reported that OS-chemoresistant cells can carry multiple drug-resistant gene phenotypes into non-drug-resistant cells through exosomes, causing non-drug-resistant OSC to develop drug resistance ${ }^{14}$. Our results are consistent with this study. The chemoresistance could relieved by inhibiting production of exosomes. Besides, Exosome could carry miRNA to achieve chemoresistance transmission. In our study, the chemoresistance of OSC could relieved by suppressing miRNA. Current studies focused on the all kinds of cell derived exosomes to regulated the proliferation, invasion and chemoresistance of OSC. LncRNA-PVT1 of stem cells can be transmit into OSC and sponged with miR-183-5p to regulate EST related gene ${ }^{15}$. Furthermore, bone marrow-derived mesenchymal stem cell-derived exosomal microRNA208a promoted the proliferation and metastasis of $\mathrm{OSC}^{16}$. Other study have found that the exosome secreted by metastatic OS cells could carry miR-675 to regulate bioactivity of OS by targeting Calnexin- 
$1^{14}$, The study also found that exosome secreted by tumor-related fibroblasts could transmit miR-1228 into OSC to promotes the proliferation and invasion of OSC by targeting SCAI ${ }^{17}$. In our study, chemoresistant OSC derived exosomes could achieve chemoresistance transmission through carrying miRNA while inhibition of miRNA could lead to suppression of chemoresistance transmission through exosomes. In thus, MiRNA-exosomes are an effective transmission route for drug resistance in OSC.

In our study, MiRNA-exosomes are an effective transmission route for drug resistance in OSC. Besides, we found miR-331 was a new miRNA which has not been reported in transmission of drug resistance. It has been reported that miR-331-3p could inhibit the proliferation and migration of colon cancer by targeting NRP $2^{18}$.Another study showed that circ-0001649 sponges with miR-331-3p, further to achieve inhibition of miR-331-3p so that the biological process of non-small cell lung cancer was suppressed ${ }^{19}$.The high expression of miR-331-3p in serum was considered to be one of the high risk factors for esophageal cancer recurrence.Studies related to miR-331 and chemotherapy in leukemia have shown that the increased expression of miR-331 may lead to poor efficacy and lower survival rate ${ }^{20}$.The above studies have shown that miR-331-3p can cause tumor recurrence and poor prognosis in some tumors. In conclusion, we found that the regulatory role of miR-331 in different tumors is not uniform. However, no relevant research reports were found on OS and miR-331. Therefore, the study on the correlation between the biological process of OS and the mechanism of drug resistance of miR-331 needs to be explored. In our study, the expression of miR-331 in OS-chemoresistant cells was higher than that in OSC. Besides, inhibition of miR-331 could relieve the chemoresistance of OSC in the level of vivo and vitro. The results in this study suggested that OS-chemoresistant cells-derived exosomal miR-331 promoted the chemoresitance of OSC while inhibition of miR-331 was an effective way to relieve the chemoresitance.

In this study, we found that exosomes of OS-chemoresistant cells could induce autophagy of OSC. It has been reported that autophagy was one of important way to lead to drug resistance ${ }^{21}$. In our study, miR331 could lead to autophagy of OSC while inhibition of miR-331 could suppress autophagy of OSC. Inhibition of miR-331-3p and miR-9-5p ameliorates Alzheimer's disease by enhancing autophagy ${ }^{22}$. It has been reported that cell proliferation was significantly reduced after transient transfection of miR-331-3p precursor in Urothelial Carcinoma ${ }^{23}$. In our study, miR-331 could promote the proliferation of OSC through exosome which derived by OSC/CDDP. Based on our results, we came up with a hypothesis, that is, miR-331 was delivered by exosomes of OS-chemoresistant cells to OSC, further to induce the autophagy and increase the cell vitality and chemoresistance of OSC. There are some limitations of this study. Since the incidence of OS is low, there is a lack of sufficient clinical samples for validation. Corresponding targeting factors and pathways also need to be further explored. Corresponding targeting factors and pathways also need to be further explored.

\section{Conclusions}

Chemoresistant OSCs-derived exosomes promote the transmission of drug resistance by carrying miR331 and inducing autophagy. Inhibition of miR-331 could effectively alleviate drug resistance of OSCs. 


\section{Abbreviations}

OS, osteosarcoma; OSC, osteosarcoma cell; miRNA, microRNA; miR-331, microRNA-331; CDDP, cisplatin; LC3, microtubule-associated protein 1 light chain 3; MDC, monodansylcadaverine; ANOVA, analysis of variance.

\section{Declarations}

\section{Ethics approval and consent to participate}

All animal experiments were performed following the approval of the Inner Mongolia Medical University Animal Ethics Committee and according to the Guidelines for the Care and Use of Laboratory Animals.

\section{Consent for publication}

Not applicable

\section{Availability of data and materials}

The analyzed datasets generated and/or analyzed during the current study are available from the corresponding author on reasonable request

\section{Competing interests}

The authors declare that they have no competing interests

\section{Funding}

The present study is supported by the National Natural Science Foundation of China (grant no. 82060488)

\section{Authors' contributions}

CYM, AQZ, YBZ, WLL, WF, RB, WHX and CSX participated in the design of the study and drafted the manuscript. RB, ZQZ, and CYM collected the data and performed the statistical analyses. CYM, ZQZ, WLL, RB and WF were major contributors to the design of this study and revised the manuscript. All authors read and approved the manuscript and agree to be accountable for all aspects of the research in ensuring that the accuracy or integrity of any part of the work are appropriately investigated and resolved.

\section{Acknowledgements}

No funding was received.

\section{References}


1. Ottaviani G, Jaffe N. The epidemiology of osteosarcoma. Cancer treatment and research. 2009;152:313.

2. Anninga JK, Gelderblom H, Fiocco M, et al. Chemotherapeutic adjuvant treatment for osteosarcoma: where do we stand? European journal of cancer (Oxford, England : 1990). 2011;47(16):2431-2445.

3. Kager L, Tamamyan G, Bielack S. Novel insights and therapeutic interventions for pediatric osteosarcoma. Future oncology (London, England). 2017;13(4):357-368.

4. Johnstone RM, Adam M, Hammond JR, Orr L, Turbide C. Vesicle formation during reticulocyte maturation. Association of plasma membrane activities with released vesicles (exosomes). The Journal of biological chemistry. 1987;262(19):9412-9420.

5. Pegtel DM, Gould SJ. Exosomes. Annual review of biochemistry. 2019;88:487-514.

6. Chicón-Bosch M, Tirado OM. Exosomes in Bone Sarcomas: Key Players in Metastasis. Cells. 2020;9(1).

7. van Niel G, D'Angelo G, Raposo G. Shedding light on the cell biology of extracellular vesicles. Nature reviews Molecular cell biology. 2018;19(4):213-228.

8. Sharma A. Chemoresistance in cancer cells: exosomes as potential regulators of therapeutic tumor heterogeneity. Nanomedicine (London, England). 2017;12(17):2137-2148.

9. Krishn SR, Singh A, Bowler N, et al. Prostate cancer sheds the av $\beta 3$ integrin in vivo through exosomes. Matrix biology : journal of the International Society for Matrix Biology. 2019;77:41-57.

10. Zhang J, Li S, Li L, et al. Exosome and exosomal microRNA: trafficking, sorting, and function. Genomics, proteomics \& bioinformatics. 2015;13(1):17-24.

11. Hayes J, Peruzzi PP, Lawler S. MicroRNAs in cancer: biomarkers, functions and therapy. Trends in molecular medicine. 2014;20(8):460-469.

12. Chen R, Wang G, Zheng Y, Hua Y, Cai Z. Drug resistance-related microRNAs in osteosarcoma: Translating basic evidence into therapeutic strategies. Journal of cellular and molecular medicine. 2019;23(4):2280-2292.

13. Qi J, Zhou Y, Jiao Z, et al. Exosomes Derived from Human Bone Marrow Mesenchymal Stem Cells Promote Tumor Growth Through Hedgehog Signaling Pathway. Cellular physiology and biochemistry : international journal of experimental cellular physiology, biochemistry, and pharmacology. 2017;42(6):2242-2254.

14. Torreggiani E, Roncuzzi L, Perut F, Zini N, Baldini N. Multimodal transfer of MDR by exosomes in human osteosarcoma. International journal of oncology. 2016;49(1):189-196. 
15. Zhao W, Qin P, Zhang D, et al. Long non-coding RNA PVT1 encapsulated in bone marrow mesenchymal stem cell-derived exosomes promotes osteosarcoma growth and metastasis by stabilizing ERG and sponging miR-183-5p. Aging. 2019;11(21):9581-9596.

16. Qin $F$, Tang $H$, Zhang $Y$, Zhang $Z$, Huang $P$, Zhu J. Bone marrow-derived mesenchymal stem cellderived exosomal microRNA-208a promotes osteosarcoma cell proliferation, migration, and invasion. Journal of cellular physiology. 2020;235(5):4734-4745.

17. Wang JW, Wu XF, Gu XJ, Jiang XH. Exosomal miR-1228 From Cancer-Associated Fibroblasts Promotes Cell Migration and Invasion of Osteosarcoma by Directly Targeting SCAl. Oncology research. 2019;27(9):979-986.

18. Zhang $\mathrm{H}$, Wang $\mathrm{R}$, Wang $\mathrm{M}$. miR-331-3p suppresses cell invasion and migration in colorectal carcinoma by directly targeting NRP2. Oncology letters. 2019;18(6):6501-6508.

19. Liu T, Song Z, Gai Y. Circular RNA circ_0001649 acts as a prognostic biomarker and inhibits NSCLC progression via sponging miR-331-3p and miR-338-5p. Biochemical and biophysical research communications. 2018;503(3):1503-1509.

20. Butrym A, Rybka J, Baczyńska D, Tukiendorf A, Kuliczkowski K, Mazur G. Expression of microRNA-331 can be used as a predictor for response to therapy and survival in acute myeloid leukemia patients. Biomarkers in medicine. 2015;9(5):453-460.

21. Zhang H, Lu B. The Roles of ceRNAs-Mediated Autophagy in Cancer Chemoresistance and Metastasis. Cancers. 2020;12(10).

22. Chen ML, Hong CG, Yue T, et al. Inhibition of miR-331-3p and miR-9-5p ameliorates Alzheimer's disease by enhancing autophagy. Theranostics. 2021;11(5):2395-2409.

23. Morita K, Fujii T, Itami H, et al. NACC1, as a Target of MicroRNA-331-3p, Regulates Cell Proliferation in Urothelial Carcinoma Cells. Cancers. 2018;10(10).

\section{Figures}




\section{miR-331-3p}

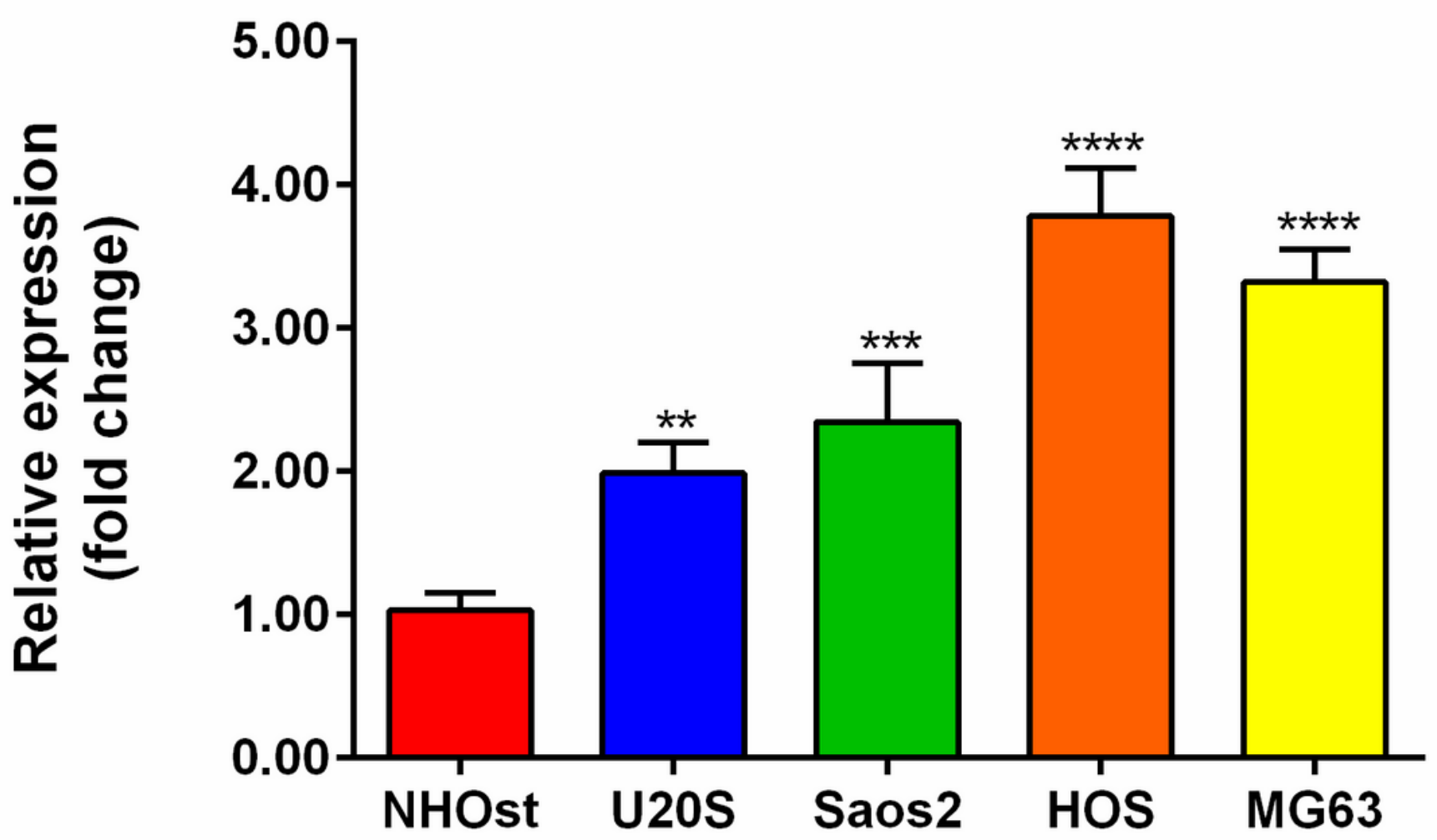

Figure 1

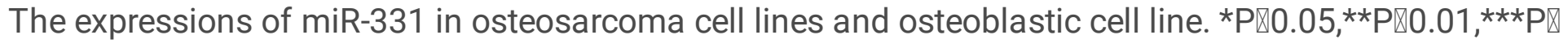
0.001 vs. the osteoblastic cell line (NHOst). 
A
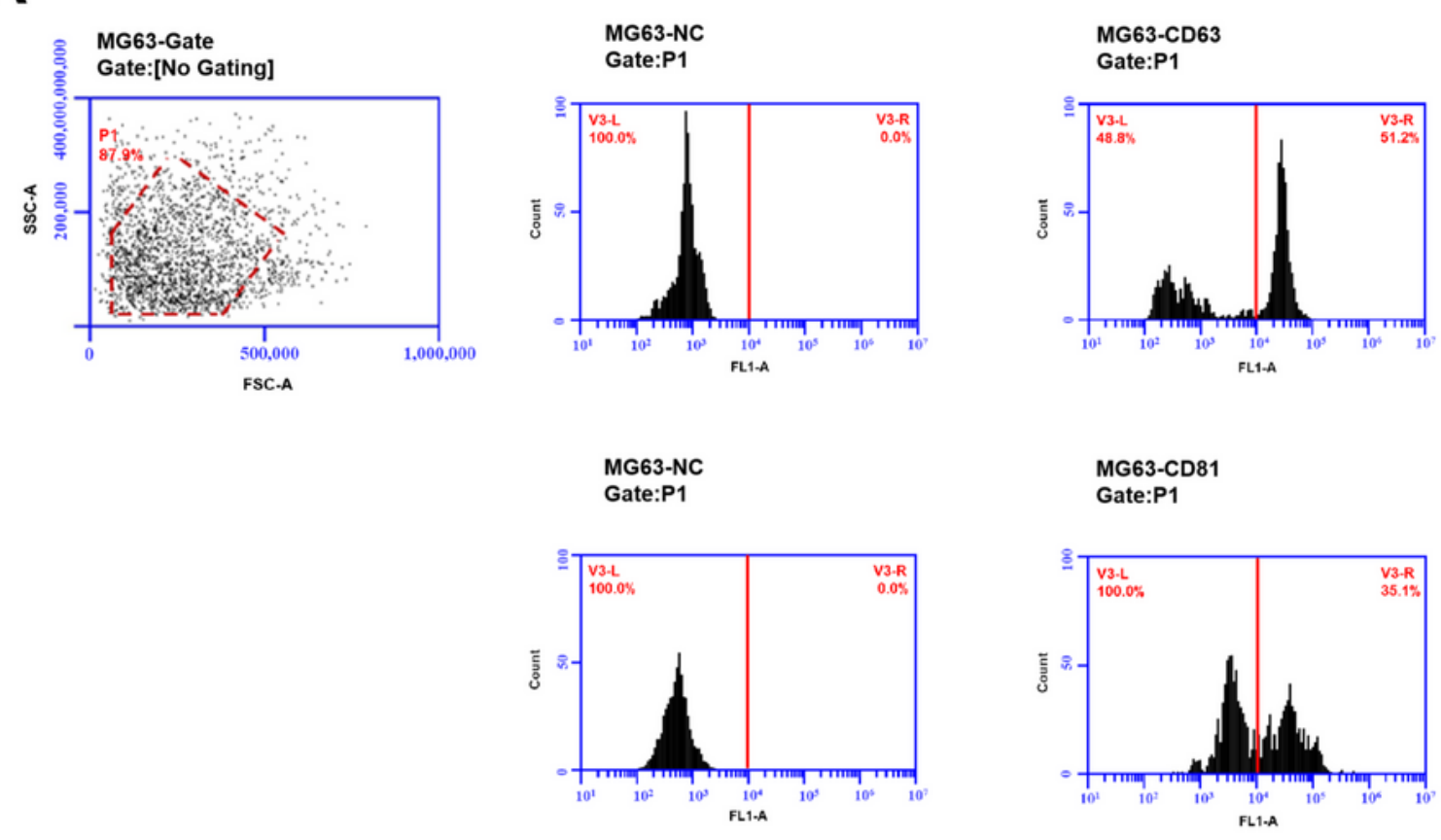

B
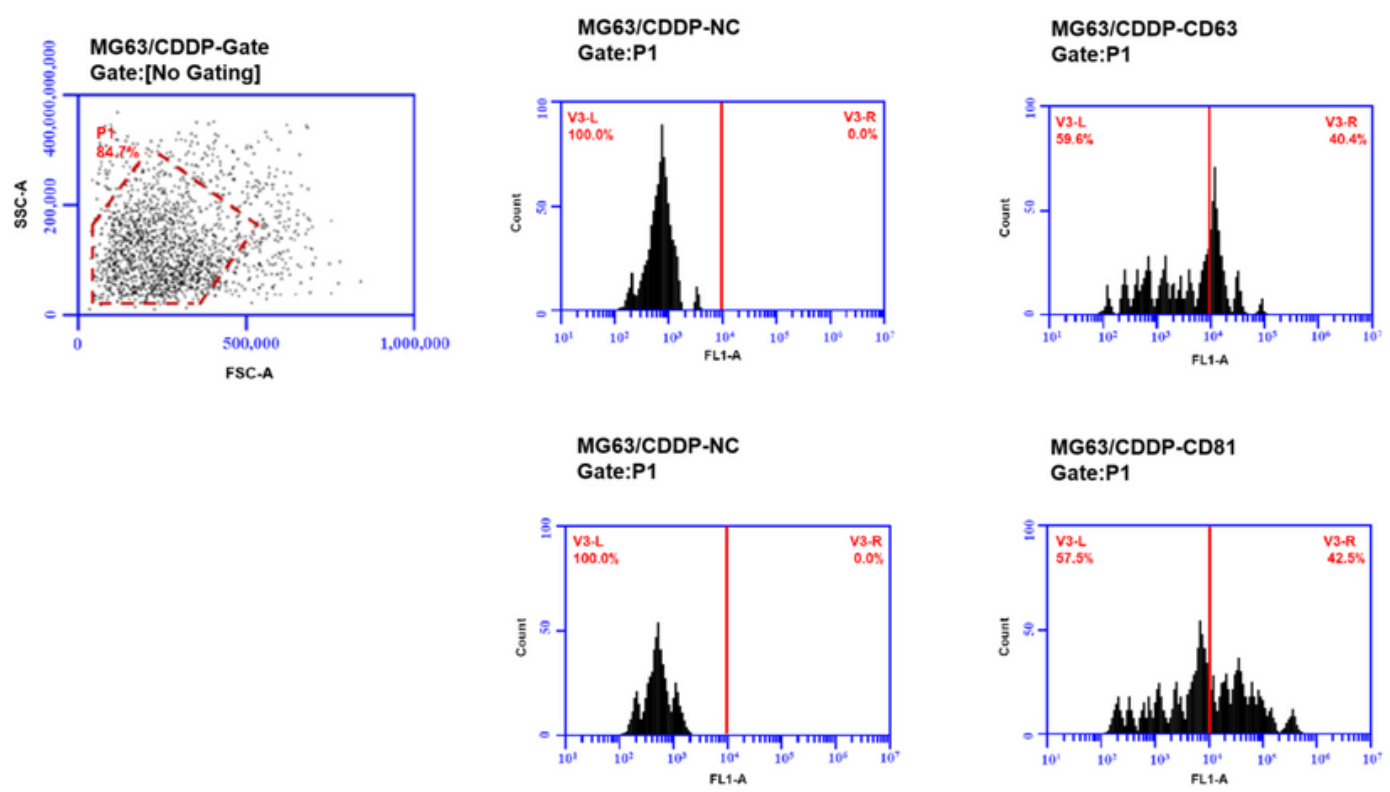

\section{Figure 2}

Flow cytometry was used to analyze the expression of CD63 and CD81 surface proteins in the supernatant exosomes of the MG63 (A) and MG63/CDDP (B) cells. 
A

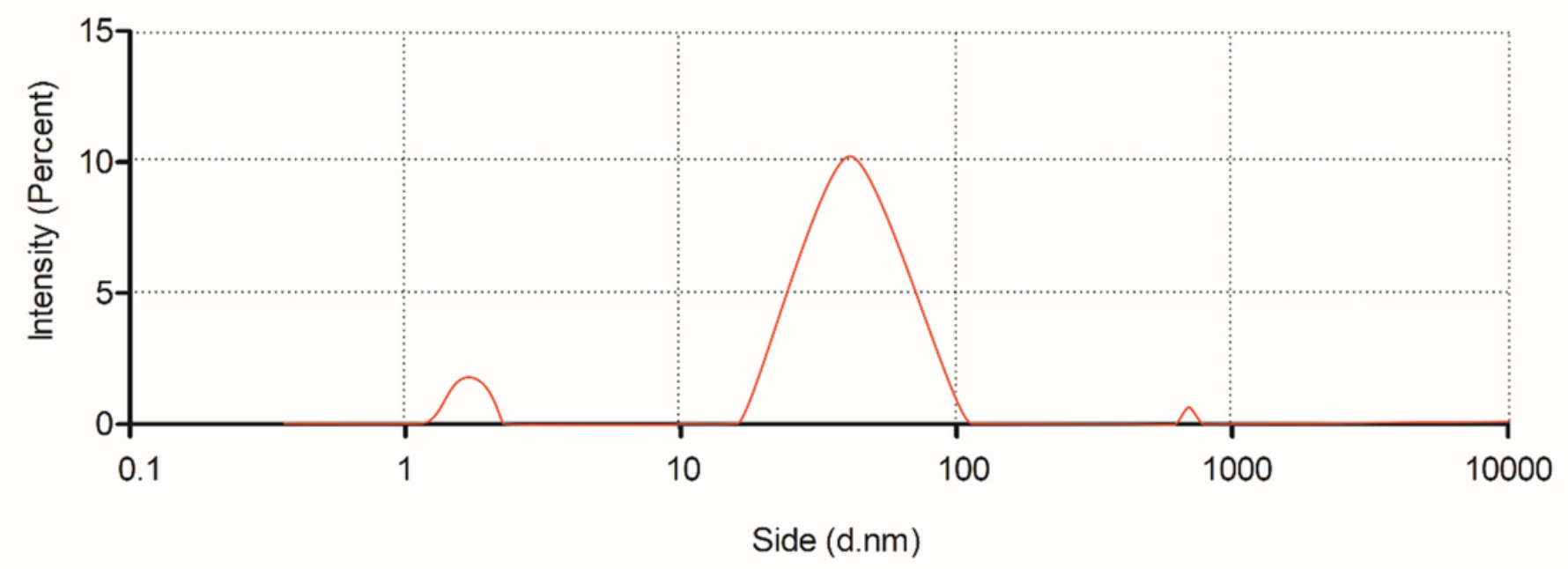

B

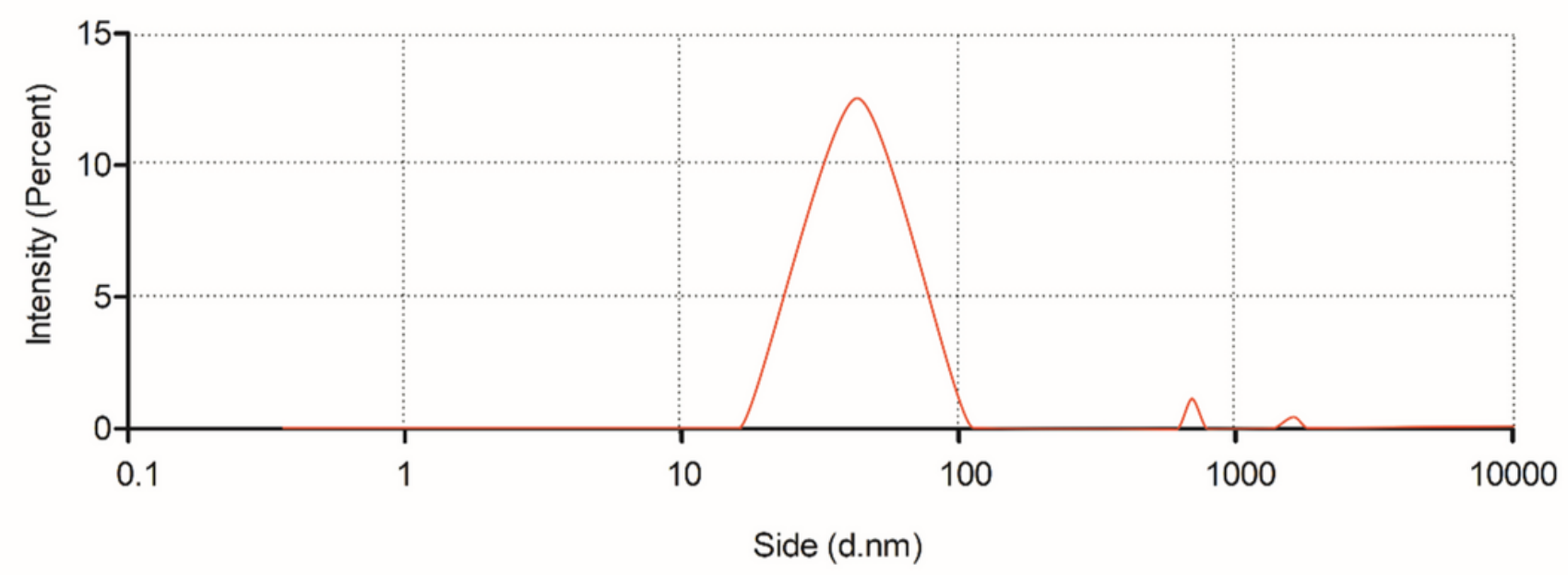

Figure 3

Granularmetric analysis of MG63 (A) and MG63/CDDP (B) exosomes 
A

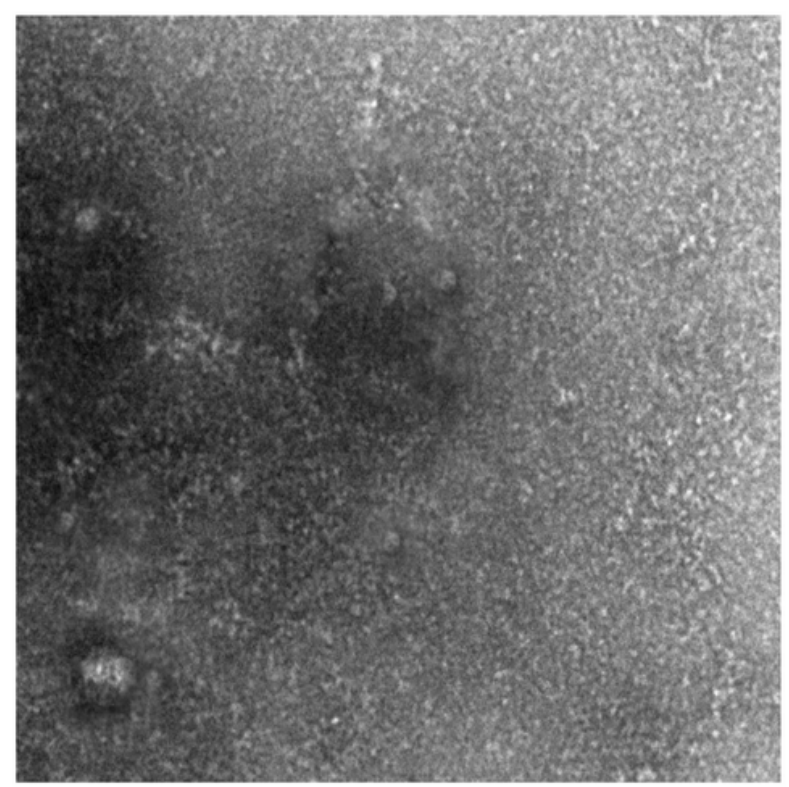

B

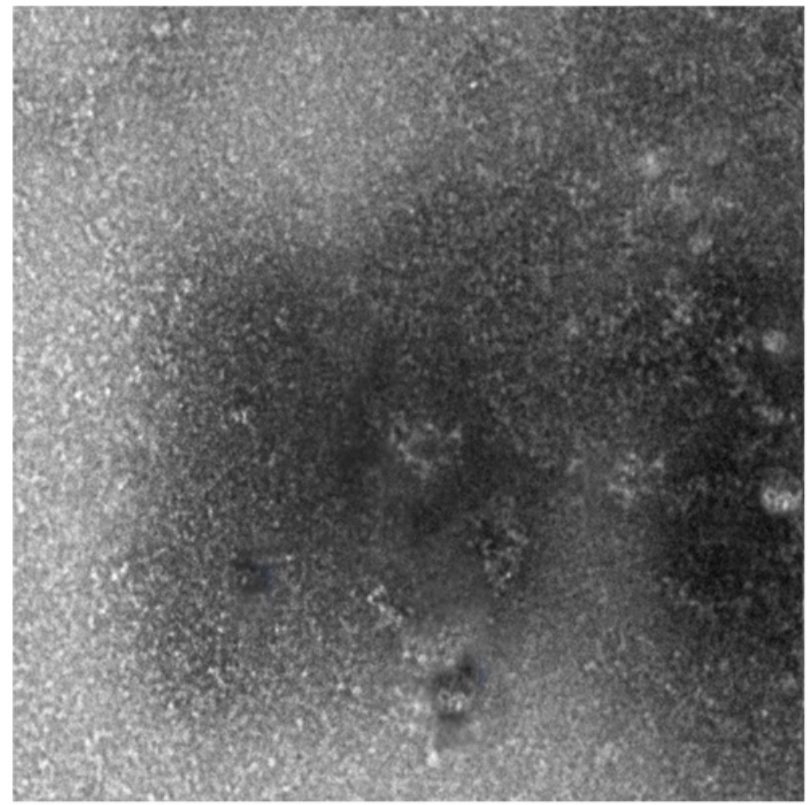

Figure 4

(A) Transmission electron microscopy image of MG63exosomes. (B)Transmission electron microscopy imageof MG63/CDDPexosomes. 
PHK26

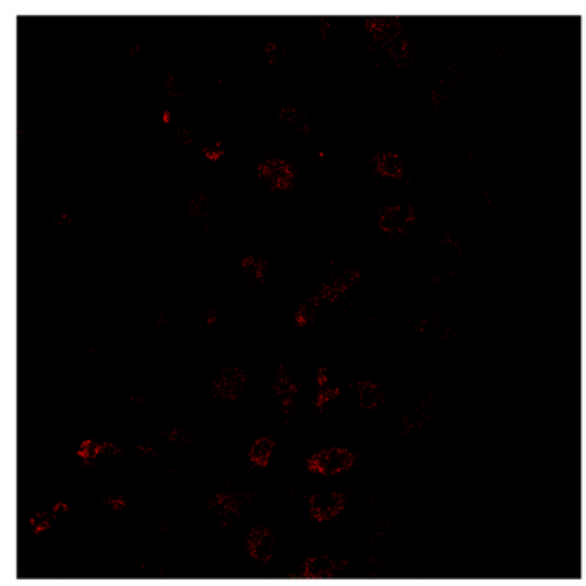

PHK26

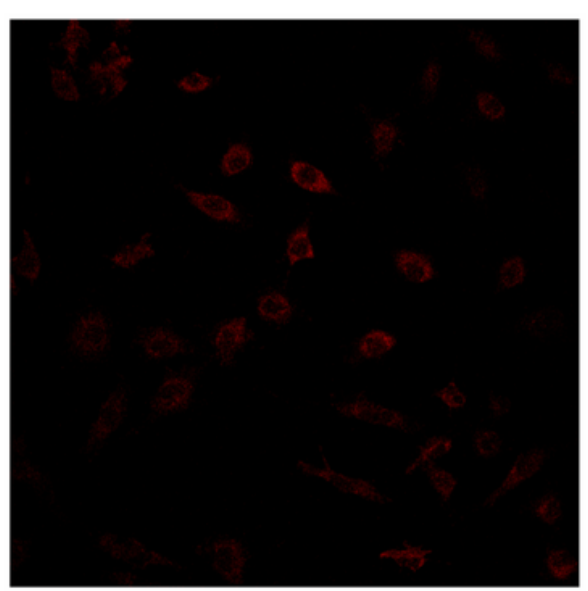

DAPI

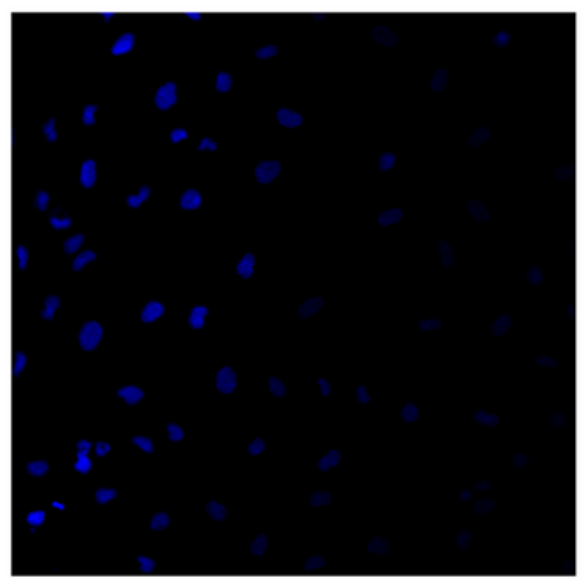

DAPI

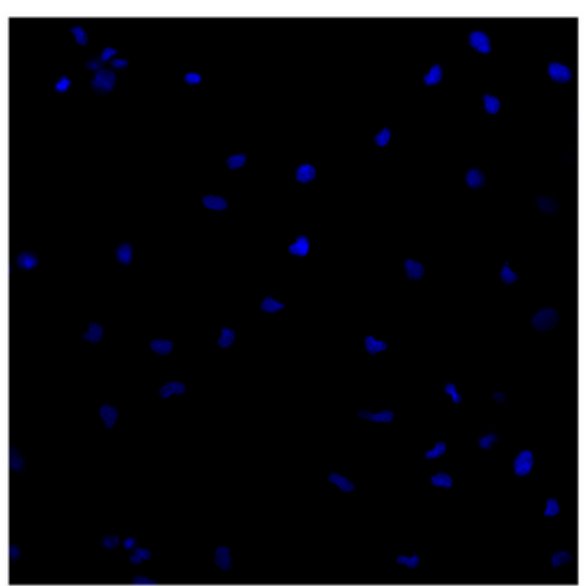

Merge

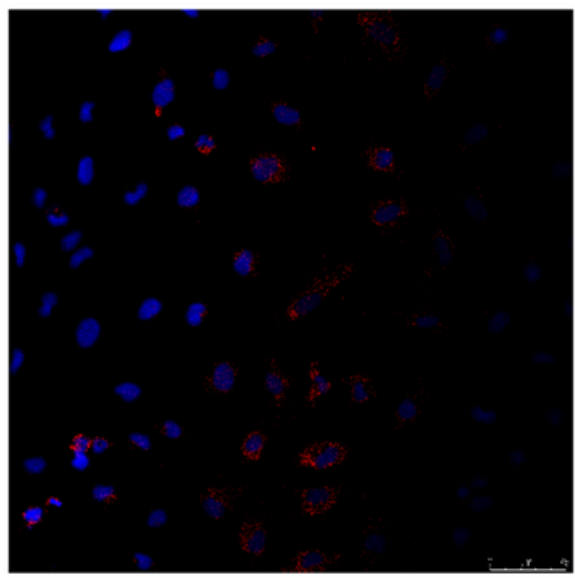

Merge

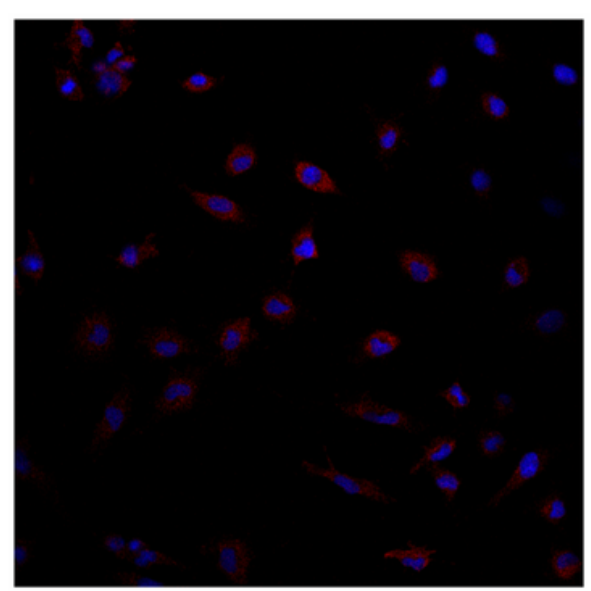

Figure 5

(A)Fluorescence image of PKH26 labeled MG63 cell-derived exosomes co-cultured with MG63 cells. (B) Fluorescence image of PKH26 labeled MG63/CDDP cell-derived exosomes co-cultured with MG63 cells. 
A

Cy3

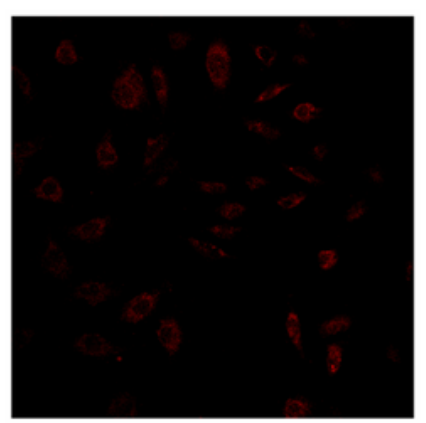

miR-NC mimics

miR-199a mimics
DAPI
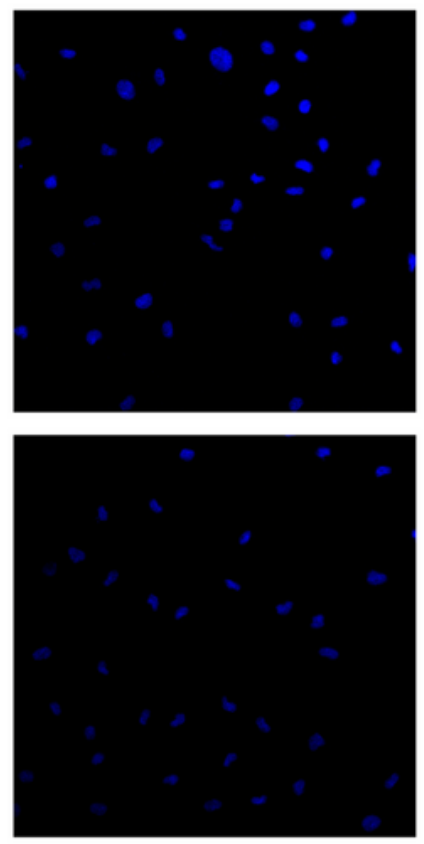

Merge
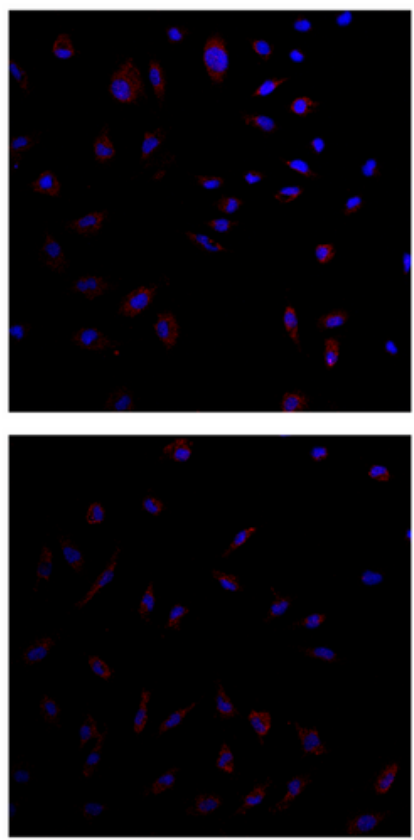

B

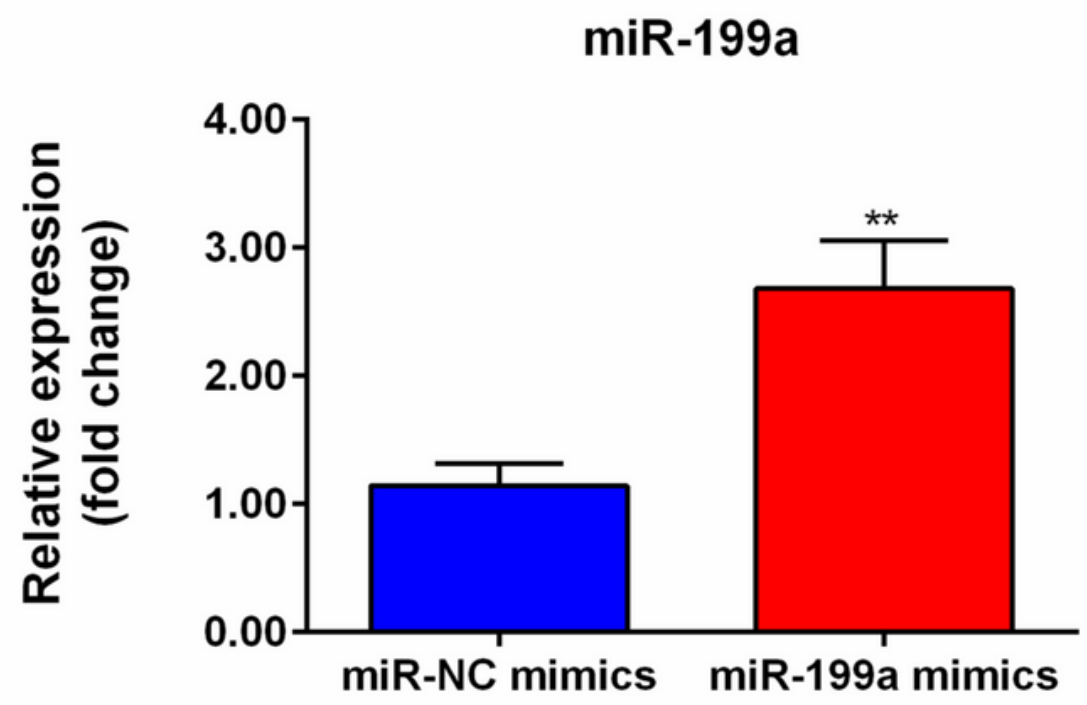

Figure 6

(A) The fluorescence of Cys in MG63 cells was observed under confocal microscope. (B)The mRNA expression level of miR-199a in lower compartment MG63 cells. ${ }^{\star \star} \mathrm{P} \otimes 0.01$ vs. the control group. 

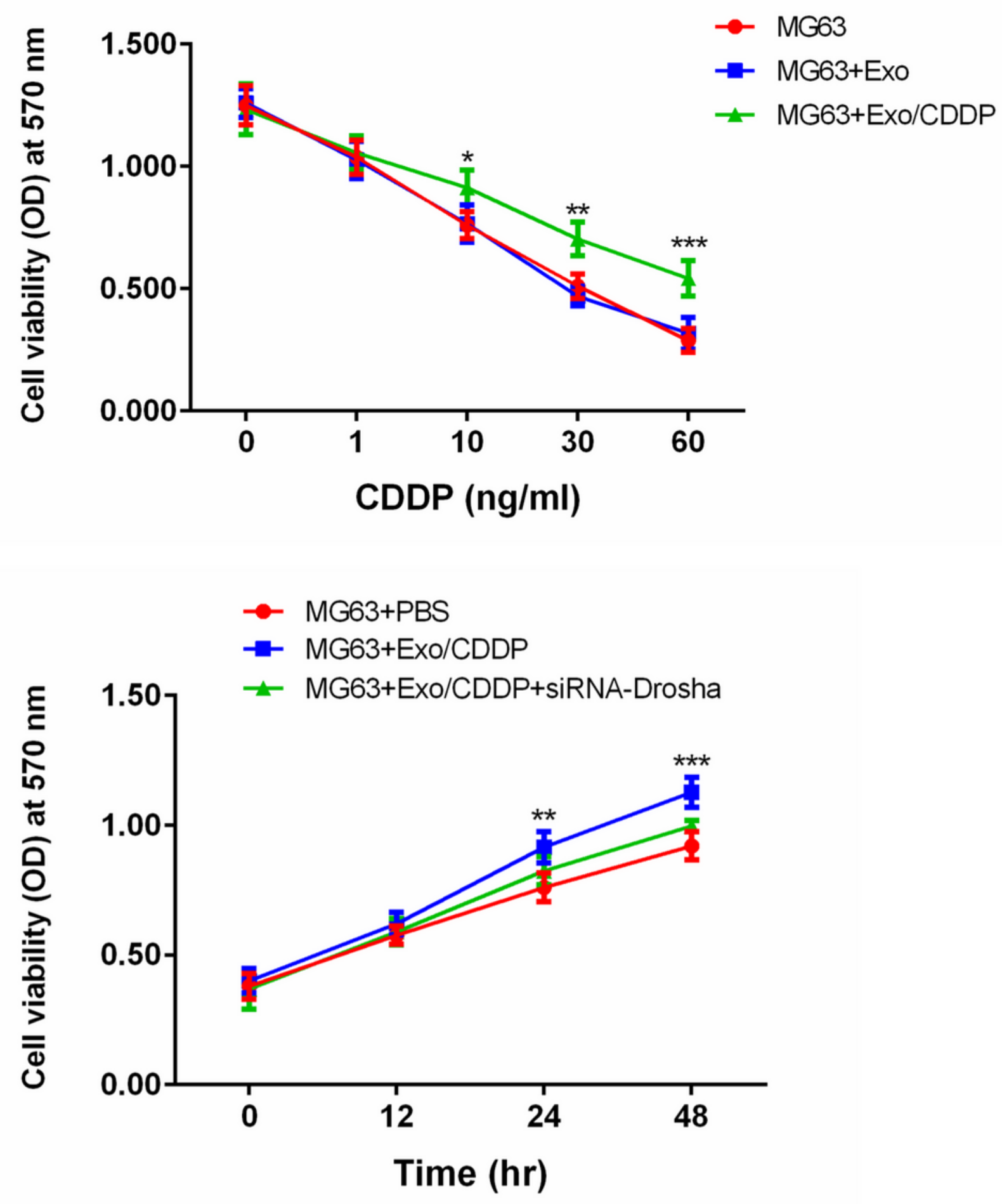

Figure 7

(A) The cell viability ofcellsingroupof MG63, MG63+Exo and MG63+Exo/CDDP at the concentrations of 10ng, 30ng and 60ng of cisplatin according to the MTT assay. (B) The cell viability of cellsingroupof MG63, MG63+Exo/CDDP and MG63+Exo/CDDP+ siRNA-Drosha at time of 0, 12h, 24h and 48h. ${ }^{*} \mathrm{P} \otimes$ 0.05 , ${ }^{\star \star} \mathrm{P} \otimes 0.01,{ }^{\star \star \star} \mathrm{P} \otimes 0.001$ vs. the MG63 control group. 


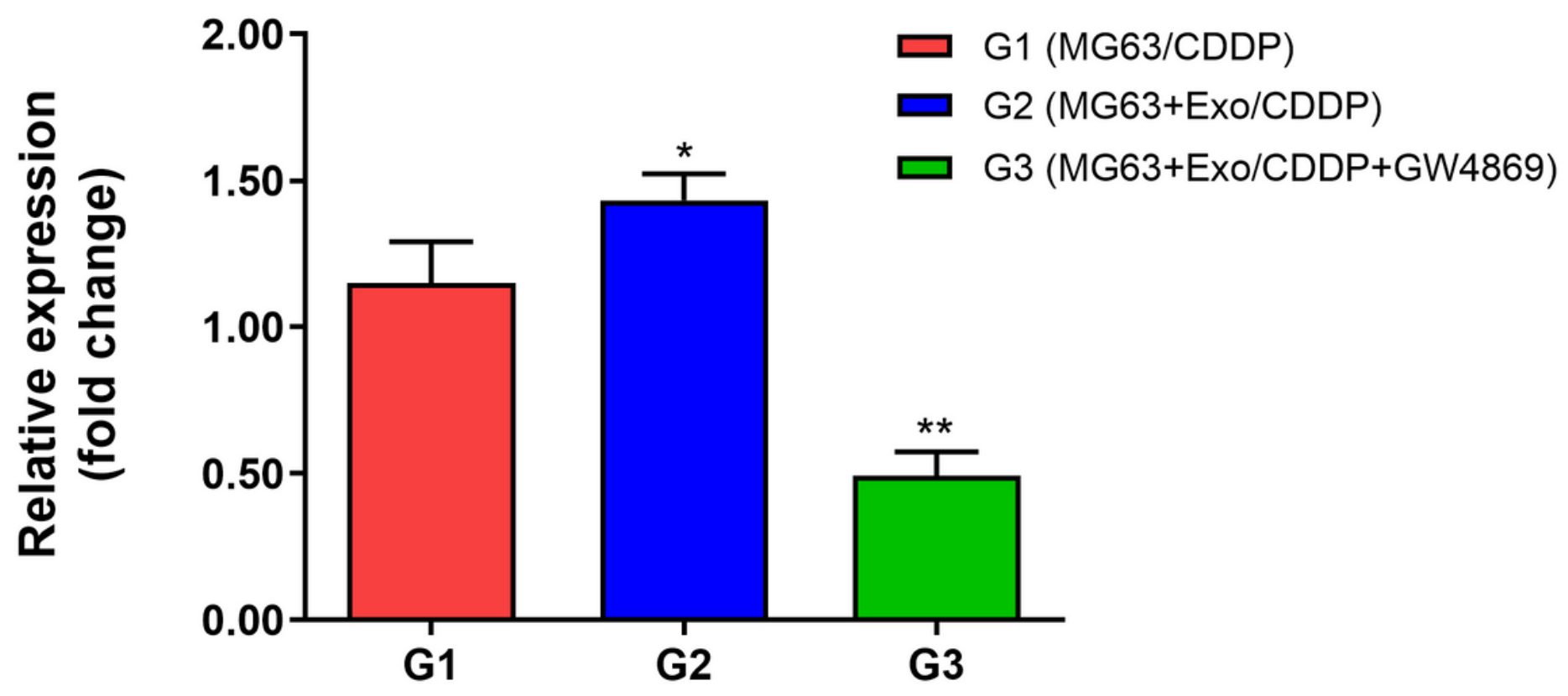

Figure 8

The expression of miR-199a in group of MG63/CDDP, MG63+Exo/CDDP and MG63+Exo/CDDP+GW4869., ${ }^{* * P ख 0.001 ~ v s . ~ t h e ~ M G 63 / C D D P ~ c o n t r o l ~ g r o u p . ~}$ 
A

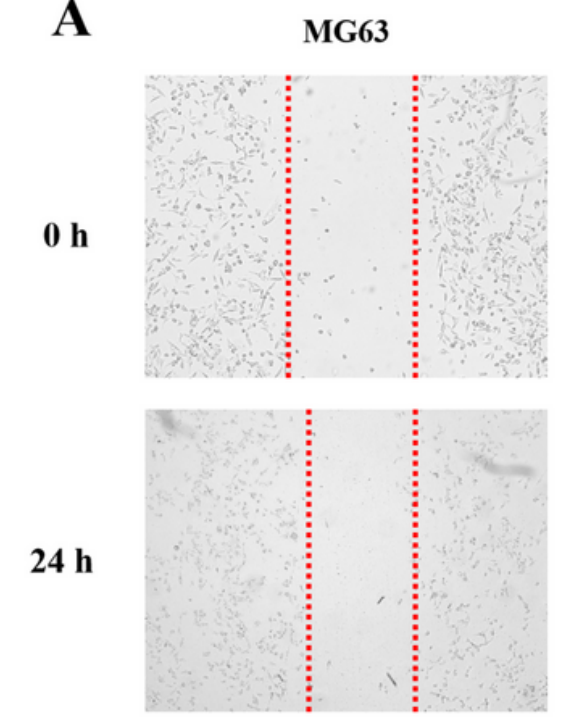

o h
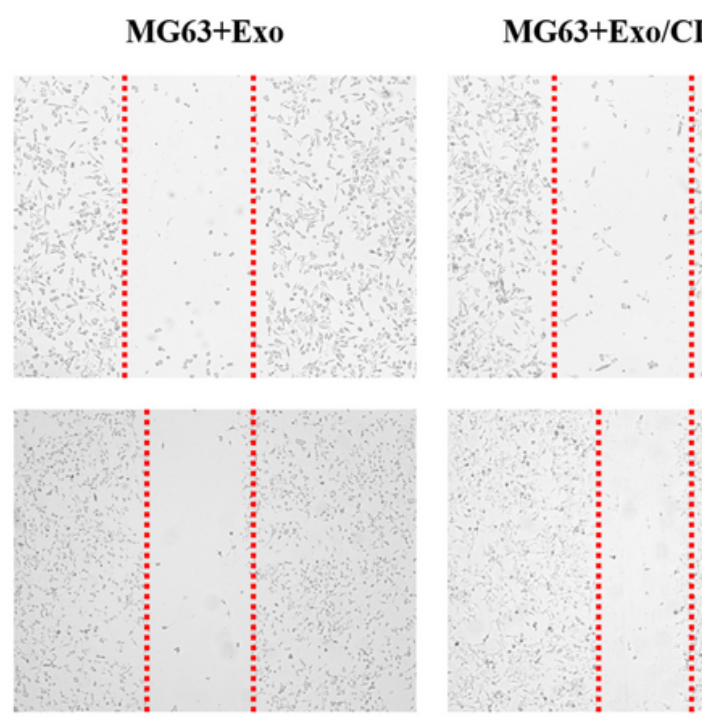

MG63/CDDP
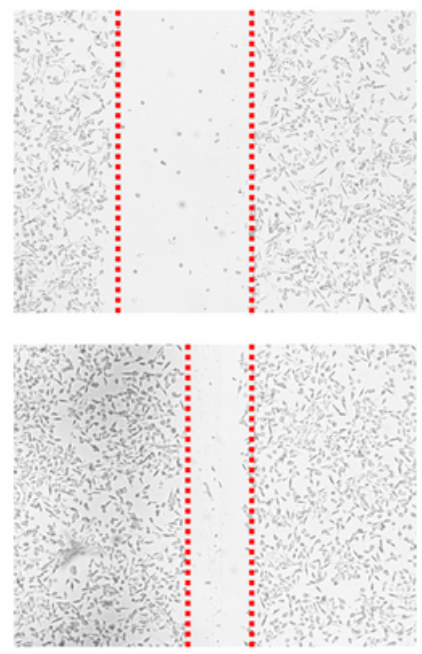

B

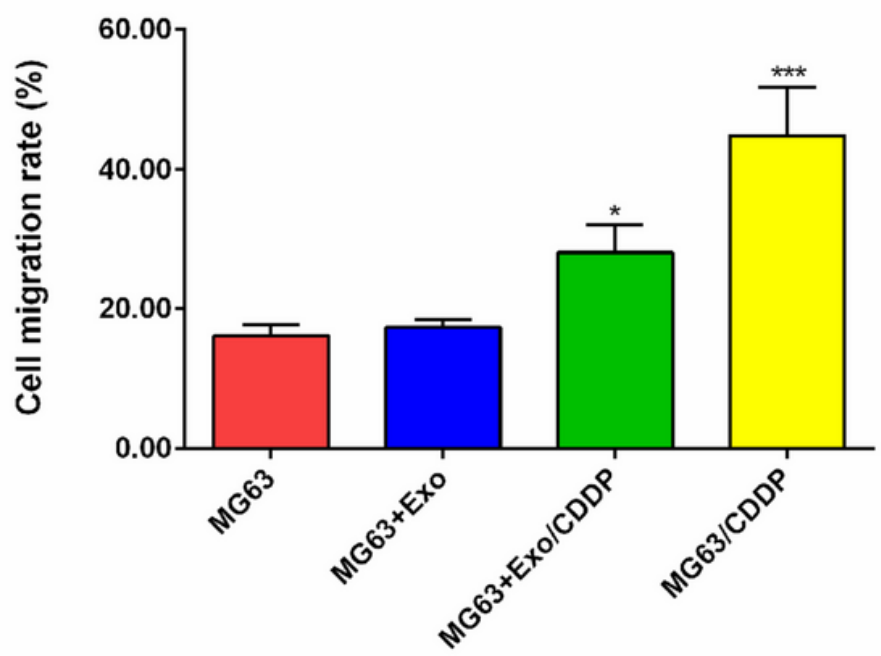

Figure 9

The ability of MG63 to migrate in groupof MG63, MG63+Exo, MG63+Exo/CDDP and MG63/CDDP. *P区 $0.05,{ }^{\star * \star} \mathrm{P} \otimes 0.001$ vs. the MG63 control group. 

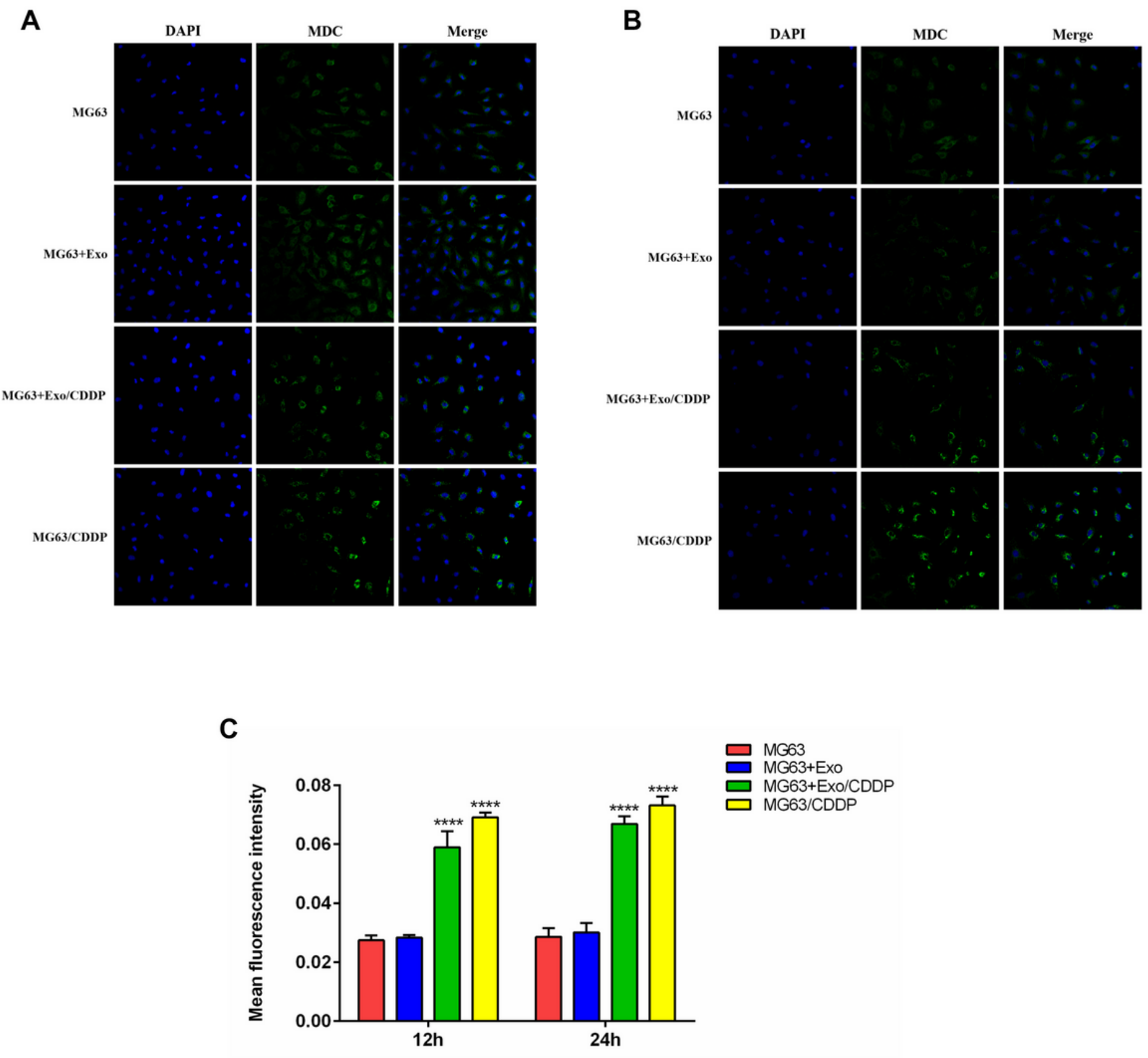

Figure 10

(A) The MDC staining to detect the autophagy in group of MG63, MG63+Exo, MG63+Exo/CDDP and MG63/CDDP. (B) The mean fluorescence intensity of each group at time of $12 \mathrm{~h}$ and $24 \mathrm{~h} .{ }^{\star} \mathrm{P} \otimes 0.05$, ${ }^{\star \star \star} \mathrm{P} \otimes$ 0.001 vs. the MG63 control group. 
A

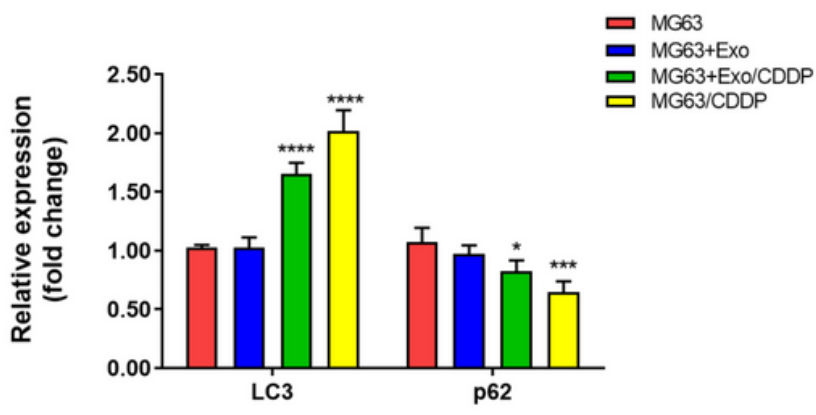

B
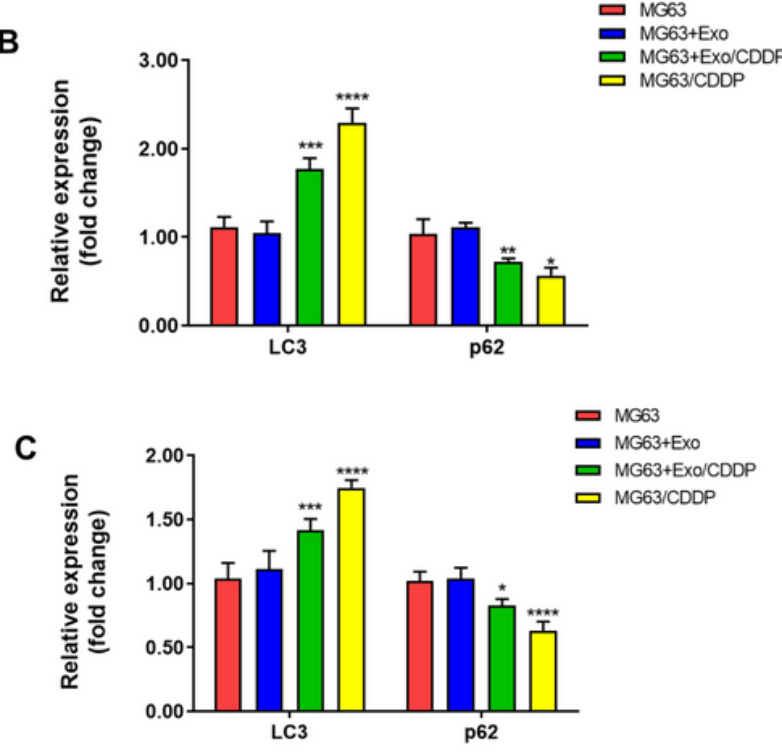
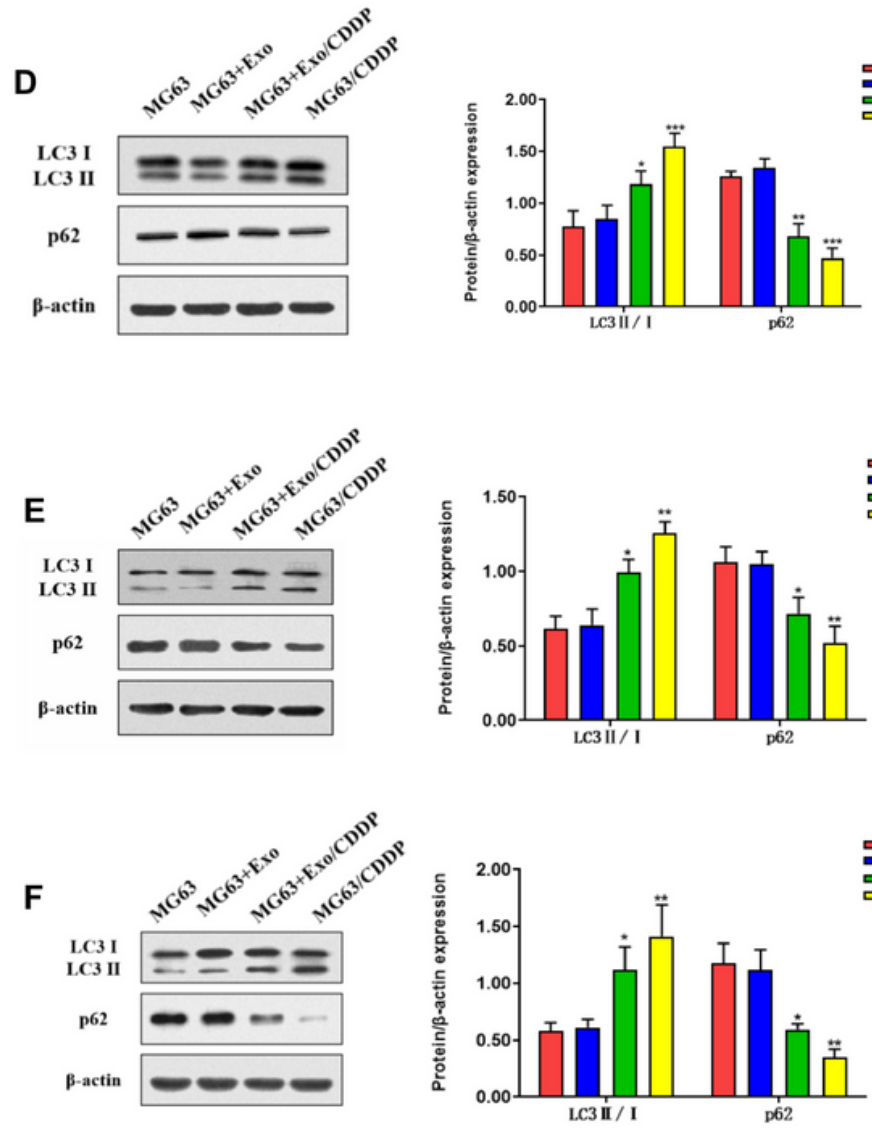

\section{Figure 11}

(A)The mRNA expression of LC3 and p62in group of MG63, MG63+Exo, MG63+Exo/CDDP and MG63/CDDP without treated by CDDP. (B) The mRNA expression of LC3 and p62in group of MG63, MG63+Exo, MG63+Exo/CDDP and MG63/CDDP treated by CDDP for 12h. (C) The mRNA expression of LC3 and p62in group of MG63, MG63+Exo, MG63+Exo/CDDP and MG63/CDDP treated by CDDP for 24h.

(D) The protein expression of LC3 and p62in group of MG63, MG63+Exo, MG63+Exo/CDDP and MG63/CDDP without treated by CDDP. (E) The protein expression of LC3 and p62in group of MG63, MG63+Exo, MG63+Exo/CDDP and MG63/CDDP treated by CDDP for $12 \mathrm{~h}$. (F) The protein expression of LC3 and p62in group of MG63, MG63+Exo, MG63+Exo/CDDP and MG63/CDDP treated by CDDP for 24h. ${ }^{*} \otimes 0.05,{ }^{\star \star} \mathrm{P} \otimes 0.01,{ }^{\star \star \star *} \mathrm{P} \otimes 0.001$ vs. the MG63 control group. 


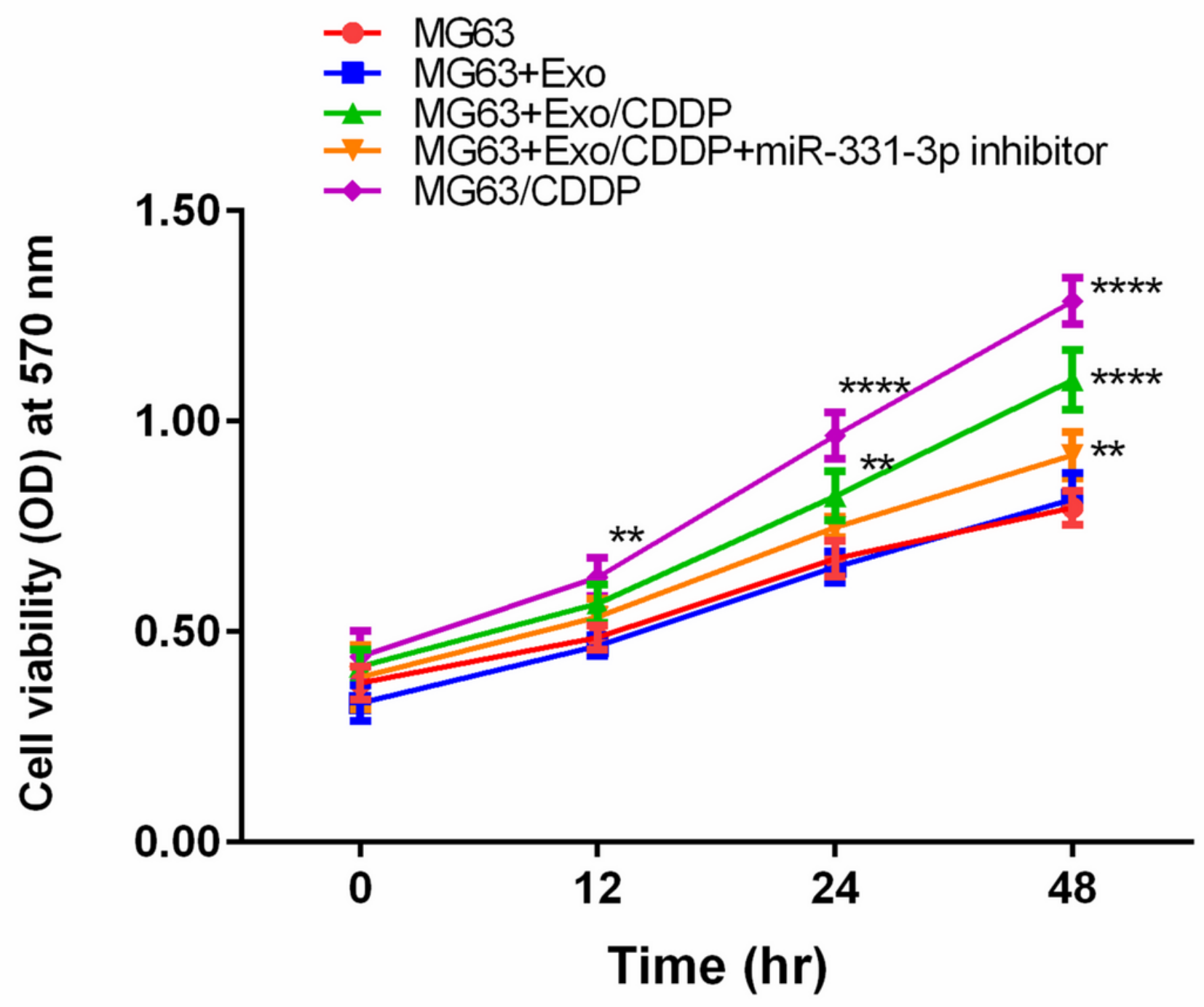

Figure 12

The cell viability of cellsingroupofMG63, MG63+Exo, MG63+Exo/CDDP, MG63+Exo/CDDP +miR-331-3p inhibitor and MG63/CDDP after treated by CDDP for $12 \mathrm{~h}, 24 \mathrm{~h}$ and $48 \mathrm{~h} .{ }^{*} \mathrm{P} \otimes 0.05$, ${ }^{\star \star} \mathrm{P} \otimes 0.01$, ${ }^{\star \star *} \mathrm{P} \rrbracket$ $0.001, \star \star * * P \llbracket 0.0001$ vs. the MG63 control group. 
A

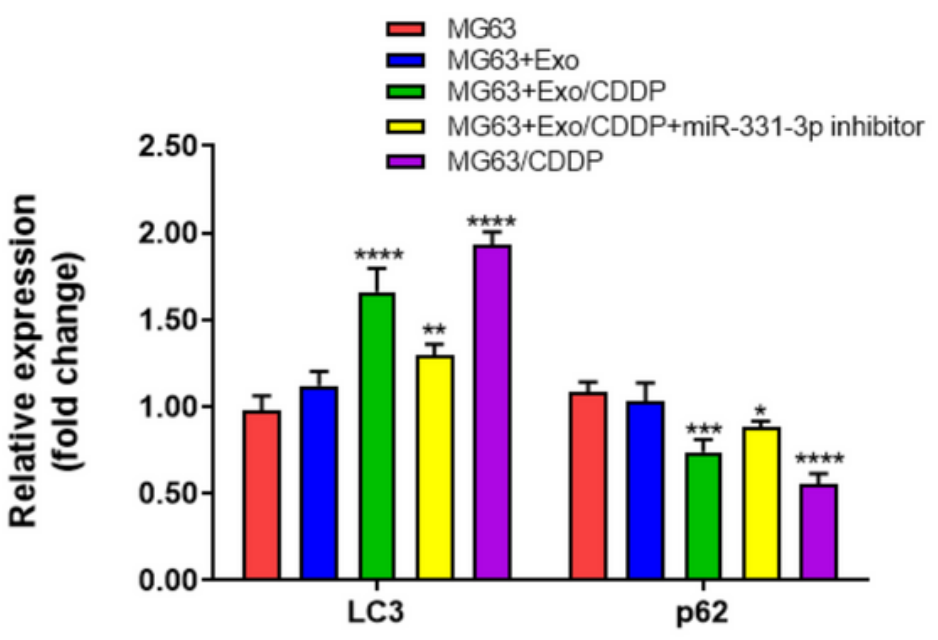

B

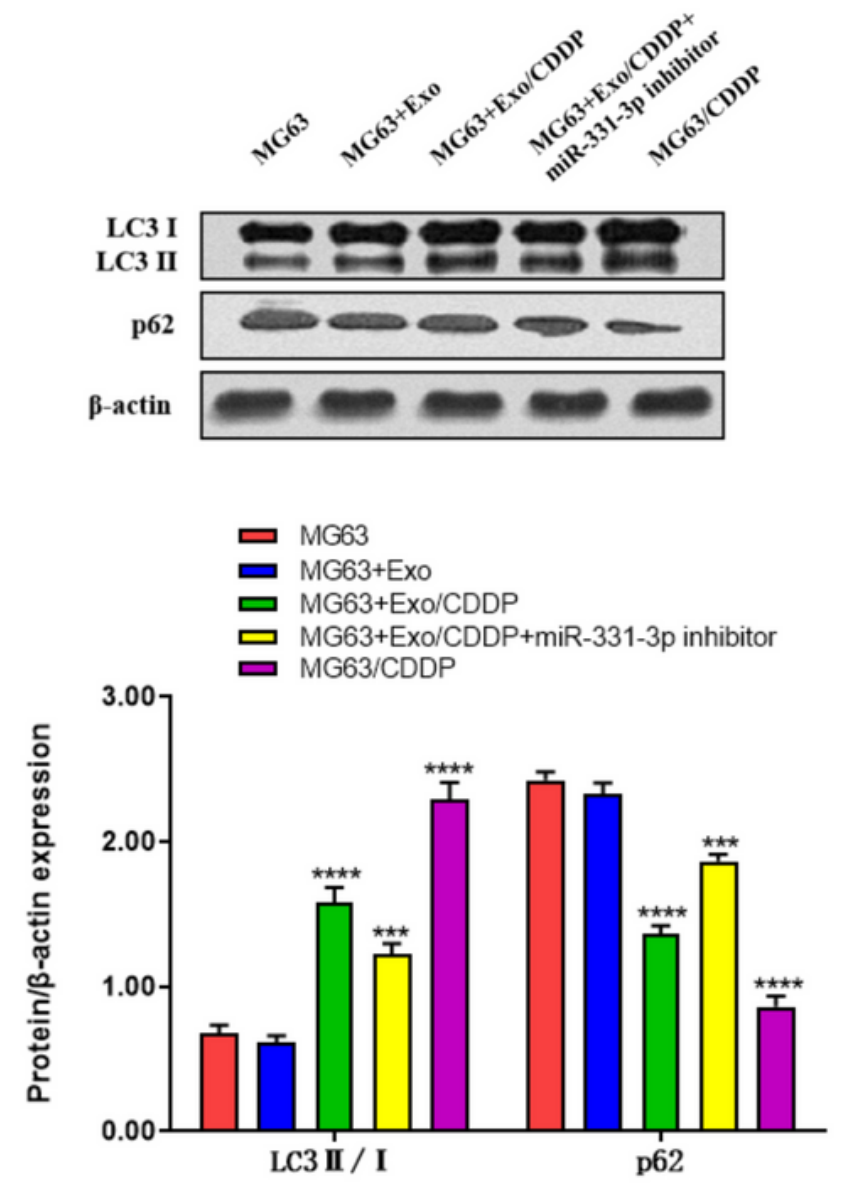

\section{Figure 13}

(A)The mRNA expression of LC3 and p62 ingroupofMG63, MG63+Exo, MG63+Exo/CDDP, MG63+Exo/CDDP +miR-331-3p inhibitor and MG63/CDDP after treated by CDDP for 24h. (B) The protein expression of LC3 and p62 ingroupofMG63, MG63+Exo, MG63+Exo/CDDP, MG63+Exo/CDDP +miR-331-

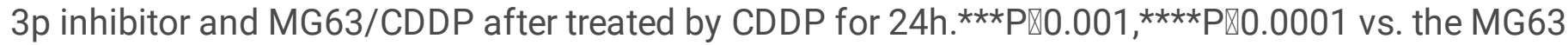
control group. 
A

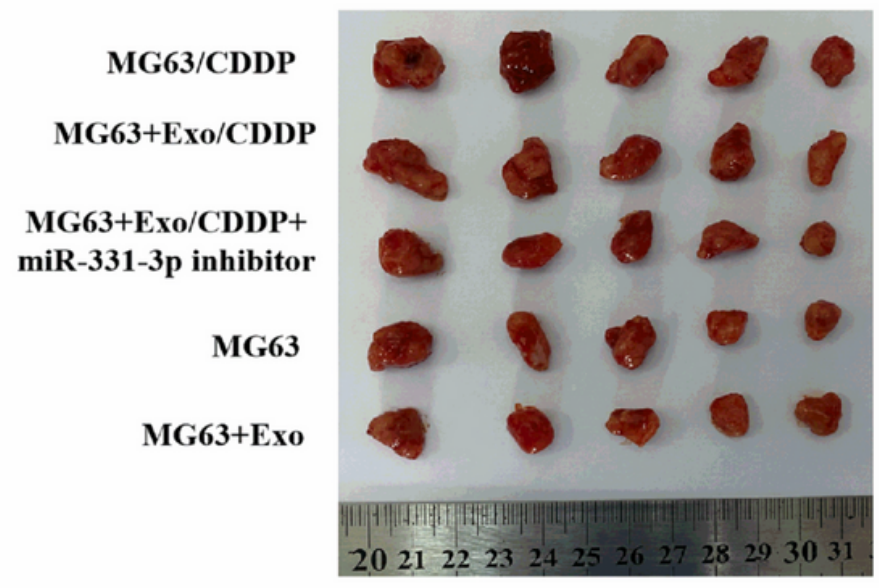

C

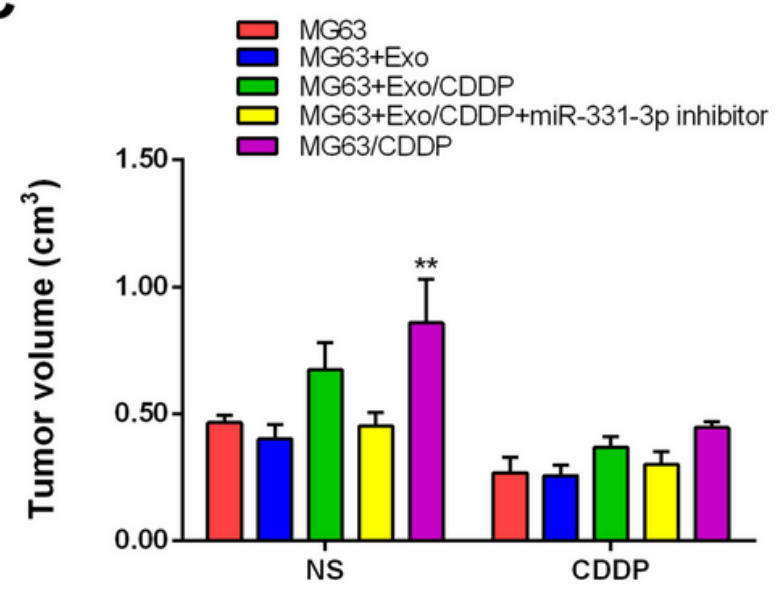

B
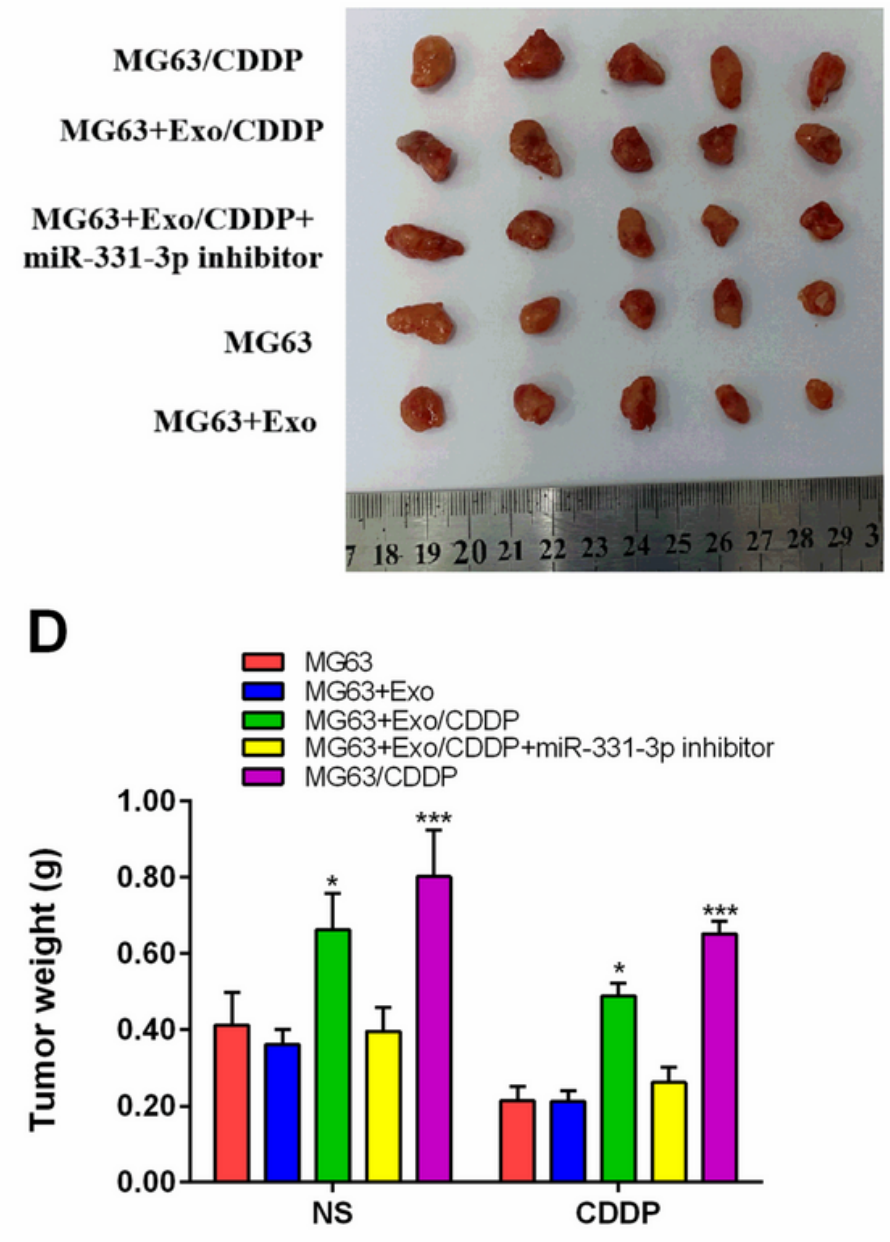

\section{Figure 14}

Results of the in vivo experiments. (A) Appearance of the tumors without treated with CDDP. (B) Appearance of the tumors treated with CDDP. (C) The tumor volume of each group. (D) The tumor weight of each group. ${ }^{\star} P \otimes 0.05,{ }^{\star \star} P \otimes 0.01,{ }^{\star \star \star * P \unrhd 0.001}$ vs. the MG63 control group. 